\title{
Optimisation des caractéristiques d'un véhicule ferroviaire en stabilité transversale et en circulation en courbe par l'algorithme génétique
}

\author{
Habib Bettaieb ${ }^{1,2, a}$ et Houcine Rejeb ${ }^{1}$ \\ 1 Laboratoire de Génie Mécanique, ENIM, Université de Monastir, 5019 Monastir, Tunisie \\ 2 École Préparatoire aux Académies Militaires, 4052 Sousse, Tunisie
}

Reçu le 3 novembre 2007, accepté le 9 juin 2008

\begin{abstract}
Résumé - Dans ce travail, une optimisation multi-objectifs est appliquée afin de trouver les valeurs optimales des paramètres de construction (paramètres d'inertie, de suspension et géométriques) d'un véhicule ferroviaire. Ces valeurs permettent d'avoir une bonne stabilité transversale lors de la circulation du véhicule en alignement et un bon comportement en courbe. Au début, on commence l'étude par une optimisation de la stabilité transversale du véhicule, ensuite, une optimisation de son comportement en courbe est faite. Les résultats ont montré qu'il y a incompatibilité entre une bonne stabilité transversale et un bon comportement en courbe. La méthode d'optimisation utilisée est la méthode des algorithmes génétiques qui est implémentée sous MATLAB.
\end{abstract}

Mots clés : Véhicule ferroviaire / stabilité transversale des véhicules / comportement en courbe / déraillement / contact roue-rail / paramètres de construction / optimisation multi-objectifs / algorithmes génétiques

\begin{abstract}
Optimization of the railway vehicle's characteristics in transverse stability and in circulation in curve using the genetic algorithm. In this work a multi objective optimization is applied in order to find the optimal values of construction parameters (inertia, suspension and geometrical parameters) of a railway vehicle. The simulation results give a good transverse stability in alignment and improve the ability to steer around a curve without a high transverse displacement and without contact flange. Under these conditions, wear of both rail and wheel is attenuated, and the lifespan of the wheel and the rail are prolonged. At the beginning, one optimizes the vehicle's transverse stability, then its behaviour in curve. The results showed that there is incompatibility between a good transverse stability and a good behaviour in curve. The method of optimization used is the method of the genetic algorithms which is implemented under MATLAB.
\end{abstract}

Key words: Simulation / railway vehicle / transverse stability of vehicle / curving performance / derailment / wheel rail contact / multi-objectives optimisation / genetic algorithm

\section{Introduction}

L'optimisation est le nom formel donné à la branche des mathématiques appliquées qui cherche à répondre à la question «Quoi de meilleur?», pour les problèmes dont la réponse peut être exprimée numériquement. Pour cela, plusieurs méthodes d'optimisation sont utilisées. Ces méthodes peuvent être classées en deux grandes catégories : les méthodes déterministes (méthode du gradient, méthode de Gauss-Seidel,...) et les méthodes stochastiques (méthode de Monte Carlo, méthode de recuit simulé, algorithmes évolutionnaires,...). Avec le

\footnotetext{
a Auteur pour correspondance:

habib.bettaieb@enim.rnu.tn
}

développement des moyens informatiques de calcul, les méthodes stochastiques sont de plus en plus utilisées. En effet, elles sont plus efficaces lorsqu'il s'agit d'optimiser des fonctions très complexes (non dérivables, bruitées, présentant plusieurs optima locaux,...) ou dont la forme est inconnue. De plus, les algorithmes évolutionnaires (algorithmes génétiques, programmation génétique, stratégies d'évolution) trouvent leur application surtout quand on essaie d'optimiser des problèmes réels (surtout industriels et scientifiques), car on est fréquemment face à des problèmes qui exigent une optimisation simultanée de plusieurs critères souvent incompatibles et contradictoires. C'est ce que l'on appelle des problèmes multi-objectifs. L'un de ces problèmes 
concerne la circulation des véhicules ferroviaires. En effet, la stabilité transversale en alignement et la sécurité du roulement en courbe sont les deux impératifs de la circulation d'un véhicule ferroviaire. Un véhicule ferroviaire circulant en alignement à une vitesse supérieure à sa vitesse critique devient instable. Aussi, en négociant une courbe, ce véhicule peut rouler avec glissement des roues par rapport aux rails, où il peut y avoir un contact entre les roues et le flanc du rail extérieur, qui peut provoquer le déraillement du véhicule. Des études ont été faites afin de montrer l'influence de la variation des paramètres de construction du véhicule sur la vitesse critique lors de sa circulation en alignement et sur son comportement en courbe [1]. Ces études ont montré qu'il y a contradiction entre une bonne stabilité transversale en alignement et un bon comportement en courbe du véhicule, d'où la nécessité d'une optimisation multi-objectifs afin de trouver les valeurs optimales des paramètres de construction du véhicule permettant une bonne stabilité transversale et un bon comportement en courbe. He et McPhee [2] ont effectué un travail d'optimisation dans ce domaine dans le but d'optimiser la stabilité transversale d'un véhicule à 17 ddl circulant en alignement. Pour cela, ils ont utilisé la méthode des algorithmes génétiques qui leur ont permis de trouver les valeurs optimales des paramètres de construction du véhicule (paramètres d'inertie, géométriques et de suspension) qui maximisent la vitesse critique du véhicule. Ils ont montré aussi que cette méthode est plus efficace que l'algorithme de Simplex dont la fiabilité diminue lorsque le nombre de variables augmente.

Le but de ce travail est l'optimisation multi-objectifs des caractéristiques mécaniques d'un véhicule ferroviaire, afin d'avoir une bonne stabilité transversale et un bon comportement en courbe. Pour cela, on commencera par présenter la méthode des algorithmes génétiques qui est la méthode d'optimisation utilisée. En deuxième lieu, on appliquera cette méthode pour l'optimisation de la stabilité transversale du véhicule. Ensuite, on l'appliquera pour optimiser son comportement en courbe. Enfin, on appliquera la même méthode pour une optimisation multiobjectifs afin de trouver les valeurs optimales des paramètres de construction du véhicule lui permettant une bonne stabilité transversale et un bon comportement en courbe.

\section{Les algorithmes génétiques}

Les algorithmes génétiques, la programmation génétique, les stratégies d'évolution, et ce que l'on appelle maintenant en général les algorithmes évolutionnaires sont des algorithmes d'optimisation stochastiques qui font partie de l'Intelligence Artificielle (IA). Les principes de base de ces algorithmes sont une transposition informatique simplifiée de la théorie d'évolution de Darwin. Les Algorithmes Génétiques (AGs) sont aujourd'hui les techniques les plus connues de ce domaine. En fait, la publication du livre de Holland en 1975 (Adaptation in Natural and Artificial Systems) marque la véritable date de naissance des AGs [3]. Ces algorithmes sont utilisés pour résoudre des problèmes dont la fonction objective (fonction du problème à maximiser ou minimiser) ne possède pas les propriétés leur permettant d'être résolus par les méthodes classiques (linéarité, continuité, dérivabilité,...) ou ceux dont la réalisation nécessite un temps de calcul considérable. Il trouve son application surtout lorsque plusieurs contraintes, parfois contradictoires, sont à satisfaire en même temps. En comparaison avec les méthodes classiques (méthodes déterministes), les AGs possèdent les avantages suivants $[2,4]$ :

- ils cherchent à travers une population de points et non un point singulier ;

- ils utilisent l'information de la fonction objective et non des dérivées ou d'autres connaissances auxiliaires ;

- ils utilisent des règles de transition probabilistes;

- ils peuvent toujours trouver l'optimum global.

La majorité des problèmes réels exige une optimisation simultanée de plusieurs critères (fonctions objectives) souvent incompatibles et contradictoires. En général, il n'y a pas une seule solution optimale à ce type de problème, mais un ensemble de solutions. Ces solutions sont optimales dans le sens qu'il n'y a pas de solutions meilleures en considérant tous les critères. C'est dans ce cas que l'utilisation des AGs vient assez naturellement à cause de leur pouvoir de générer plusieurs solutions en une seule itération.

\subsection{Processus de l'algorithme génétique simple}

En général, le problème à optimiser est formulé de la façon suivante :

$$
\left\{\begin{aligned}
\text { maximiser ou minimiser } & f(X) \\
\text { sous contraintes : } & g_{j}(X) \leq 0 \\
& X \in \Omega \\
& f \in \Omega^{\prime}
\end{aligned}\right.
$$

où $f$ est la fonction objective, $X$ est la ou les variables dont on veut trouver la ou les valeurs optimales, $\Omega$ l'espace de recherche de $X$ et $\Omega^{\prime}$ l'espace de recherche de $f$.

L'algorithme génétique est une technique probabiliste qui manipule des populations successives. Il transforme une population d'individus (une valeur de $X$ est un individu), chacun avec une valeur d'aptitude associée, en une nouvelle génération de la population, en utilisant le principe darwinien de la survie et de la reproduction du plus convenable et les analogues des opérateurs génétiques naturels tels que le croisement et la mutation.

Il y a quatre étapes préparatoires principales exigées pour utiliser l'algorithme génétique conventionnel pour résoudre un problème [5] :

(1) la représentation spécifique du problème;

(2) la fonction d'aptitude;

(3) les paramètres et les variables pour commander l'algorithme;

(4) un critère d'arrêt. 
Une condition préalable pour résoudre un problème avec l'algorithme génétique est que la représentation spécifique soit suffisante pour exprimer une solution au problème. Cette représentation structure le problème pour le résoudre par l'algorithme génétique et détermine les opérateurs génétiques à utiliser. Trouver une représentation qui facilite la solution d'un problème par l'algorithme génétique exige souvent une perspicacité considérable et un bon jugement du problème. Chaque point dans l'espace de recherche d'un problème est codé, en utilisant une représentation spécifique du problème, en tant que chaîne de caractères de longueur constante (c-à-d. comme chromosome), par exemple en binaire (0 et 1 ), en symboles (A, B, C,...), etc. Aussi, les individus peuvent être des valeurs réelles ou entières. Michalewicz a montré que la représentation réelle des individus est plus efficace en temps de calcul et en précision du résultat que la représentation binaire [6]. L'algorithme génétique essaie de trouver la meilleure (ou au moins une très bonne) solution au problème en reproduisant génétiquement la population des individus à travers un certain nombre de générations.

Le processus évolutionnaire est conduit par la fonction d'aptitude. Cette fonction assigne une valeur d'aptitude à chaque individu de la population. La fonction d'aptitude doit être entièrement définie dans le sens qu'elle soit capable d'évaluer n'importe quel individu dans n'importe quelle génération de la population. La nature de cette fonction dépend du problème. Les paramètres primaires pour commander l'algorithme génétique sont la taille de population, et le nombre maximum des générations. En outre, il y a un certain nombre de paramètres secondaires concernant les opérateurs génétiques qui doivent être indiqués afin d'exécuter l'algorithme génétique. Chaque exécution de l'algorithme génétique exige la spécification d'un critère d'arrêt pour décider quand terminer l'exécution. Le critère d'arrêt pour une exécution de l'algorithme génétique consiste habituellement à satisfaire un attribut de succès spécifique du problème, ou accomplir un nombre maximal indiqué de générations. Une fois que les quatre étapes préparatoires, pour lancer l'algorithme génétique, ont été accomplies, l'algorithme génétique peut être exécuté. La figure 1 présente un organigramme de l'algorithme génétique simple.

Ainsi, en suivant la boucle représentée par la figure 1, on passe de génération en génération, jusqu'à obtenir la solution optimale. Dans la suite, on détaillera les étapes principales de cette boucle.

\subsection{Optimisation multi-objectifs par les AGs}

Généralement, un problème multi-objectif à optimiser est formulé de la façon suivante :

$$
\left\{\begin{array}{cl}
\min / \max \quad y & =f(X) \\
& =\left(f_{1}(X), f_{2}(X), \ldots, f_{n}(X)\right) \\
\text { sous contraintes : } \quad X=\left(x_{1}, x_{2}, \ldots, x_{m}\right) \in \Omega & \\
y & =\left(y_{1}, y_{2}, \ldots, y_{n}\right) \in \Omega^{\prime}
\end{array}\right.
$$

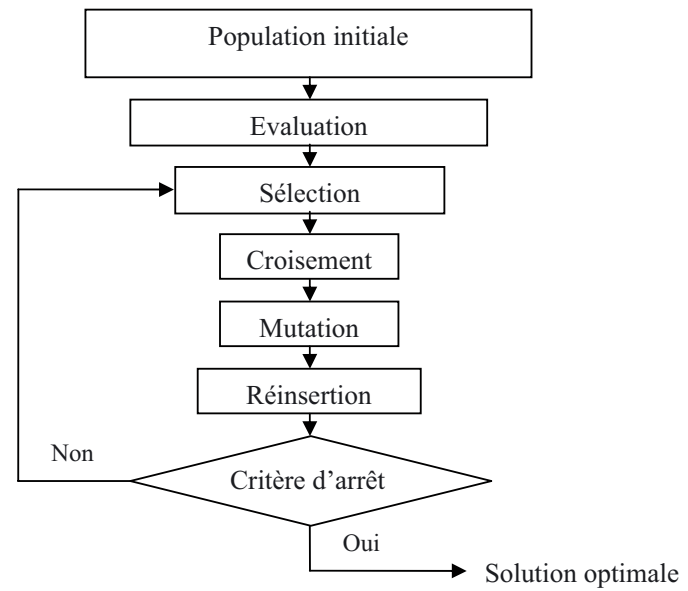

Fig. 1. Organigramme de l'algorithme génétique simple.

Pour résoudre ce type de problèmes, deux approches ont été utilisées au début :

- la méthode de la somme pondérée $[7,8]$ qui consiste à associer un poids $w_{i}$ à chaque fonction objective $f_{i}$ et à les sommer pour obtenir une seule fonction objective :

$$
s(x)=\sum_{i=1}^{n} w_{i} f_{i}(x)
$$

avec, $\sum_{i=1}^{n} w_{i}=1$

- optimiser l'une des fonctions $f_{i}$ et mettre les autres sous forme de contraintes $[7,8]$.

Des reproches ont été faits à ces deux méthodes à cause de la difficulté d'estimation des poids et l'homogénéisation des différentes fonctions en une seule unité de mesure (qui est parfois impossible [4]) pour la première, et le choix de la fonction à optimiser et des limites pour les fonctions mises comme contraintes pour la deuxième. De plus, les deux approches consistent à rendre le problème multi-objectif en un problème simple à une seule fonction objective dont l'optimisation conduit à une seule solution. Or, pour les problèmes multi-objectifs, il y a des solutions que l'on ne peut comparer sans donner de priorités aux divers critères $f_{i}$ : ce sont les solutions optimales selon le principe de dominance de Pareto $[4,7,8]$. On dit que ces solutions sont les solutions non dominées. Selon ce principe, une solution $x_{1}$ domine une solution $x_{2}$ (on écrit $x_{1}>x_{2}$ ) si et seulement si :

$$
\begin{aligned}
\forall j & \in\{1, \ldots, n\} f_{j}\left(x_{1}\right) \geq f_{j}\left(x_{2}\right) \\
\text { et } \exists i & \in\{1, \ldots, n\} f_{i}\left(x_{1}\right)>f_{i}\left(x_{2}\right)
\end{aligned}
$$

L'ensemble des solutions non dominées est appelé le front de Pareto $[4,7,8]$. Prenons l'exemple d'un problème multiobjectifs à deux fonctions objectives. Supposons qu'on veut minimiser les deux fonctions et qu'on ait les solutions suivantes :

$\mathrm{A}=(2,10)\left(f_{1}, f_{2}\right)$

$\mathrm{B}=(4,6) ; \mathrm{C}=(8,4) ; \mathrm{D}=(9,5) ; \mathrm{E}=(7,8)$ 


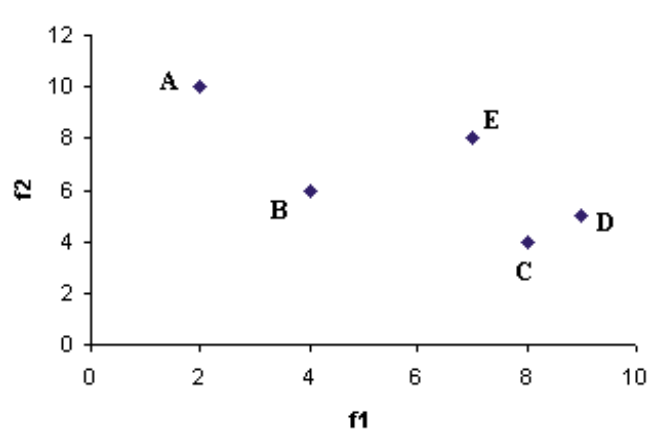

Fig. 2. Solutions de l'exemple.

Ces solutions sont représentées sur la figure 2.

La solution E est dominée par la solution B car $4<7$ et $6<8$. La solution $\mathrm{D}$ est dominée par $\mathrm{C}$ car $8<9$ et $4<5$. Pour chacune des solutions A, B, et C, il n'y a pas de meilleure pour les deux fonctions $\left(f_{1}, f_{2}\right)$. L'ensemble $\{\mathrm{A}, \mathrm{B}, \mathrm{C}\}$ constitue le front de Pareto.

Puisque les AG simples peuvent générer plusieurs solutions en une seule itération, alors, on les utilise pour trouver le front de Pareto d'un problème multi-objectif. Il suffit d'une légère modification du processus de l'AG simple [3] : la sélection doit être adaptée de façon à pousser la population vers le front de Pareto (c'est-à-dire changer la méthode de l'attribution de la valeur d'aptitude (fitness) aux individus), tout en maintenant une bonne diversité de la population pour assurer un bon échantillonnage du front de Pareto (éviter la concentration des solutions en un seul point du front de Pareto). Pour la préservation de la diversité, plusieurs méthodes ont été utilisées, parmi lesquelles on cite la méthode de partage des ressources (« sharing », [3, 4, 9]), la méthode de similarité ( crowding scheme », $[3,4,8]$ ) et la méthode de reproduction restreinte (« restricted mating », $[4,8]$ ) qui sont les plus utilisées. Les deux premières méthodes sont inspirées du phénomène naturel de création de niches écologiques (sous-populations stables).

Pendant la recherche des solutions optimales, de bonnes solutions peuvent être perdues. Pour remédier à ce problème, une stratégie d'élitisme est indispensable $[8,10,11]$. En général, deux approches élitistes sont utilisées : l'une consiste à copier un certain nombre des solutions non dominées de la génération $t$ à la génération $t+1$, l'autre consiste à maintenir une population secondaire, appelée archive, dans laquelle on copie les solutions non dominées à chaque génération. Cette archive peut être utilisée comme un stockage externe indépendant du processus d'optimisation ou intégrée dans l'algorithme pour participer aux opérations génétiques. Souvent, la taille de l'archive est maintenue constante. Pour cela, des critères doivent être définis pour le choix des solutions à insérer et à éliminer de l'archive. Le critère de dominance est le plus utilisé : les nouvelles solutions non dominées remplacent les solutions de l'archive qui les dominent. Un autre critère de densité peut être utilisé en plus pour garder une bonne diversité des solutions contenues dans l'archive.
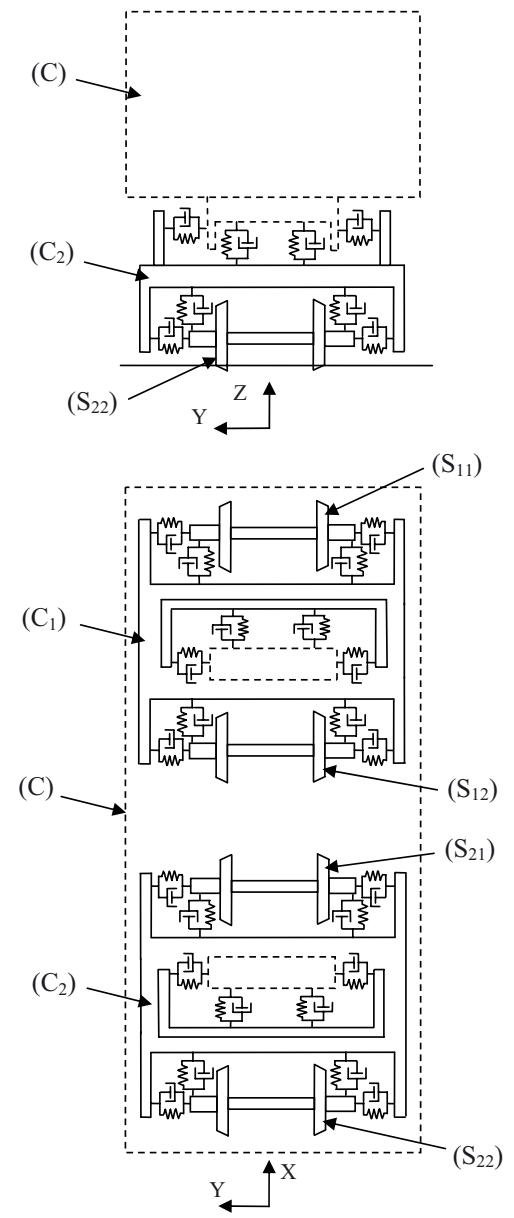

Fig. 3. Modèle du véhicule pour la simulation en alignement droit.

\section{Optimisation de la stabilité transversale d'un véhicule ferroviaire circulant en alignement}

Dans cette partie, on appliquera la méthode des algorithmes génétiques afin d'optimiser la stabilité transversale d'un véhicule ferroviaire circulant en alignement.

\subsection{Modèle du véhicule et hypothèses}

Le système mécanique représentant le véhicule ferroviaire est montré par la figure 3 .

Ce système est composé de sept solides supposés rigides et indéformables :

- 1 solide principal ou caisse $(C)$;

- 2 solides intermédiaires ou châssis de bogies $\left(C_{k}\right)(k=$ 1,2), supposés identiques ;

- 4 solides inférieurs ou essieux $\left(S_{k i}\right)(i=1,2)$, supposés identiques, en contact avec la voie.

Ces différents solides sont reliés entre eux par des systèmes élastiques supposés linéaires. Chaque essieu est lié au châssis de bogie correspondant par la suspension 
primaire et chaque châssis de bogie est lié à la caisse par la suspension secondaire. Chaque composant de la suspension est constitué d'un ressort et d'un amortisseur montés en parallèle dans les trois directions. Le véhicule présente une symétrie longitudinale et transversale des inerties et des élasticités. Dans cette étude, on s'intéresse uniquement aux mouvements de la dynamique transversale. Ce véhicule est supposé rouler à une vitesse constante $V$ le long d'une voie rectiligne de bonne qualité. Les valeurs nominales des différents paramètres d'inertie, de suspension et géométriques sont indiquées dans l'annexe A. L'étude est faite dans un repère $R_{0}=\left(O_{0}, x_{0}, y_{0}, z_{0}\right)$ qui est en translation rectiligne uniforme de vitesse $V$ par rapport à un repère galiléen $g=\left(O_{\mathrm{g}}, x_{\mathrm{g}}, y_{\mathrm{g}}, z_{\mathrm{g}}\right)$. Pour tous les solides, les mouvements considérés sont le déplacement latéral, le lacet (rotation autour de l'axe $z$ ) et le roulis (rotation autour de l'axe $x$ ). Ces mouvements sont notés comme suit :

- la caisse $(C): \bar{Y}, \bar{\alpha}, \bar{\theta}$

- les châssis de bogies $\left(C_{k}\right): Y_{k}, \alpha_{k}, \theta_{k}$

- les essieux $\left(S_{k i}\right): y_{k i}, \alpha_{k i}, \theta_{k i}$.

\subsection{Modèle dynamique}

Le modèle dynamique du véhicule est obtenu à partir des équations de Lagrange :

$$
\frac{\mathrm{d}}{\mathrm{d} t}\left(\frac{\partial E}{\partial \dot{q}_{i}}\right)-\frac{\partial E}{\partial q_{i}}+\frac{\partial U}{\partial q_{i}}=F_{q_{i}}
$$

avec $E$ : énergie cinétique du véhicule, $U$ : énergie potentielle, $F_{q_{i}}$ : force généralisée, $q_{i}$ : coordonnée généralisée (système à $17 \mathrm{ddl}$ ) :

$$
\begin{array}{r}
\{q\}=\left[\bar{Y}, \bar{\alpha}, \bar{\theta}, Y_{1}, \alpha_{1}, \theta_{1}, y_{11}, \alpha_{11},\right. \\
\left.y_{12}, \alpha_{12}, Y_{2}, \alpha_{2}, \theta_{2}, y_{21}, \alpha_{21}, y_{22}, \alpha_{22}\right]^{T}
\end{array}
$$

Les mouvements considérés pour les essieux $\left(S_{k i}\right)$ sont le ballant $y_{k i}$, le roulis $\theta_{k i}$ et le lacet $\alpha_{k i}$. La théorie de l'étude géométrique du contact entre le rail et la roue fournit la relation entre le roulis $\theta_{k i}$ et le ballant $y_{k i}$ [12] :

$$
\theta_{k i}=\Gamma y_{k i} \text { avec } \Gamma=\frac{\gamma_{0}}{e_{0}-r_{0} \gamma_{0}}
$$

Les forces généralisées sont tirées de l'expression de la puissance dissipée par les amortisseurs et les actions de contact rail et roue. Ces dernières sont représentées par la figure 4.

Le torseur de ces actions au point de contact $I_{k i j}$ s'écrit comme suit :

$$
\left\{\begin{array}{c}
X_{k i j} x_{0}+T_{k i j} u\left(\gamma_{j}\right)+N_{j} u\left(\gamma_{j}+\frac{\pi}{2}\right) \\
M_{k i j} u\left(\gamma_{j}+\frac{\pi}{2}\right)
\end{array}\right\}
$$

où $j$ est l'indice de la roue, $j=1$ : roue gauche dans le sens de l'avancement, $j=2$ : roue droite dans le sens de

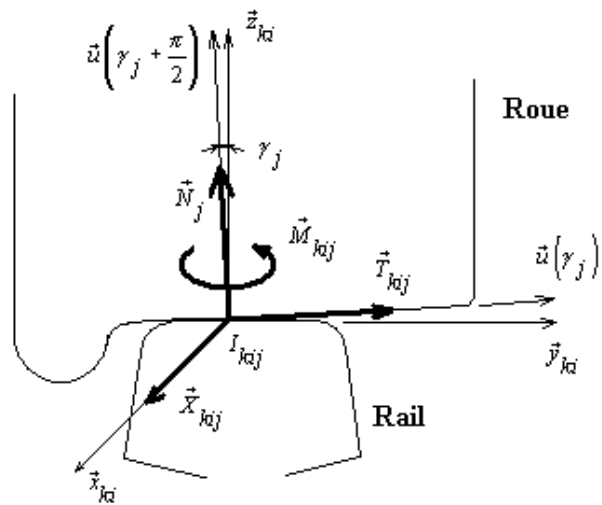

Fig. 4. Actions de contact rail-roue.

l'avancement, $X_{k i j}, T_{k i j}$ et $M_{k i j}$, étant appelées forces de pseudo-glissement, sont données par la théorie de Kalker :

$$
\begin{aligned}
X_{k i j} & =-C_{11} \nu_{k i j} \cdot x_{0} \\
T_{k i j} & =-C_{22} \nu_{k i j} \cdot u\left(\gamma_{j}\right)-C_{23} \phi_{k i j} \\
M_{k i j} & =C_{23} \nu_{k i j} \cdot u\left(\gamma_{j}\right)-C_{33} \phi_{k i j}
\end{aligned}
$$

$C_{11}, C_{22}, C_{23}$ et $C_{33}$ sont les coefficients de Kalker, ils dépendent des modules d'élasticité transversale et des coefficients de Poisson des matériaux de la roue et du rail, ainsi que des dimensions de l'ellipse de contact calculées à partir de la théorie de Hertz.

$\nu_{k i j}$ est le glissement réduit au point de contact $I_{k i j}$

$\phi_{k i j}$ est le spin au point $I_{k i j}$.

Les équations de mouvement obtenues à partir des équations de Lagrange peuvent être mises sous la forme matricielle suivante :

$$
\mathrm{M}\{\ddot{q}\}+\mathrm{C}\{\dot{q}\}+\mathrm{K}\{q\}=\{0\}
$$

avec $\mathrm{M}$ : matrice de masse, $\mathrm{C}$ : matrice d'amortissement, $\mathrm{K}$ : matrice de rigidité, $\{q\}$ : vecteur de coordonnées généralisées.

Le système (11) peut être stable ou instable, et peut être résolu par l'utilisation des équations d'état :

$$
\{\dot{Y}\}=\mathrm{A}\{Y\}
$$

avec $:\{Y\}=\left\{\begin{array}{l}\{q\} \\ \{\dot{q}\}\end{array}\right\}$

$$
A=\left[\begin{array}{cc}
0 & \mathrm{I}_{17 \times 17} \\
-\mathrm{M}^{-1} \mathrm{~K} & -\mathrm{M}^{-1} \mathrm{C}
\end{array}\right]
$$

La solution du système (12) est la suivante :

$$
\{Y\}=\sum_{i=1}^{34}\left\{A_{i}\right\} e^{\lambda_{i} t}
$$

avec $\lambda_{i}$ : les valeurs propres de $\mathrm{A}$, elles sont deux à deux conjuguées, $\left\{A_{i}\right\}$ : les modes propres de $\mathrm{A}$.

Ainsi, le système n'est stable que si toutes les valeurs propres sont à partie réelle négative. 


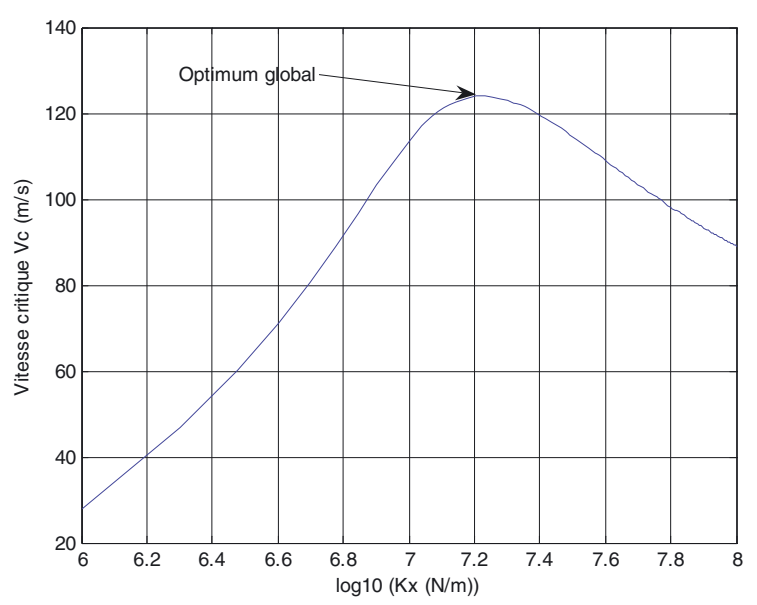

Fig. 5. Variation de $V_{\text {c }}$ en fonction de $K_{x}$.

\subsection{Stabilité du véhicule}

La matrice A dépend des caractéristiques de construction du véhicule : les paramètres d'inertie, les paramètres de suspension et les paramètres géométriques. Elle est aussi fonction de la vitesse $V$ du véhicule. En faisant augmenter cette vitesse, pour des valeurs de paramètres de construction constantes, on remarque qu'à une certaine valeur de $V$, la partie réelle de deux valeurs propres devient positive. Le comportement du véhicule est instable; ce phénomène est connu sous le nom d'instabilité de lacet (en anglais : « hunting ») $[2,12,14]$ qui est caractérisé par une augmentation de la fréquence d'oscillation et une diminution de l'amortissement. L'amplitude de ce phénomène est de plus en plus importante en augmentant la vitesse. Ainsi, le véhicule roulant à la vitesse à laquelle la partie réelle des deux valeurs propres est nulle, est à la limite de la stabilité. Cette vitesse, notée $V_{\mathrm{c}}$, est appelée vitesse critique. Alors, en faisant varier les valeurs des paramètres de construction du véhicule, on obtient différentes valeurs de $V_{\text {c }}$. Un programme de calcul sous MATLAB permet d'obtenir la figure 5 qui montre l'influence de la variation de la rigidité $K_{x}$ (les autres paramètres ayant leurs valeurs nominales) sur la vitesse critique $V_{\mathrm{c}}$.

On constate que cette courbe a un optimum global qui correspond à une vitesse critique de $123 \mathrm{~m} . \mathrm{s}^{-1}$ pour une rigidité $K_{x o p}=1,5 \times 10^{7} \mathrm{~N} . \mathrm{m}^{-1}$. Pour les valeurs de $K_{x}$ inférieures à cette valeur, les ressorts sont relativement souples, ce qui engendre une atteinte plus rapide de la vitesse critique. Au-delà de cette valeur, les ressorts sont très rigides $\left(K_{y}\right.$ rigide aussi), le système devient comme étant un bloc ce qui augmente les chocs et par suite l'atteinte rapide de la vitesse critique. Les autres paramètres de suspension dont la variation influe considérablement sur la vitesse critique sont $K_{y}, \bar{K}_{x}, \bar{K}_{y}$. La figure 6 montre l'influence de la variation de $\bar{K}_{y}$.

De même, on remarque que la courbe de $V_{\text {c }}$ possède un optimum global en faisant varier chaque variable. On remarque aussi que, pour la variation de $V_{\text {c }}$ en fonction de $\bar{K}_{y}$, une légère augmentation de la valeur optimale de

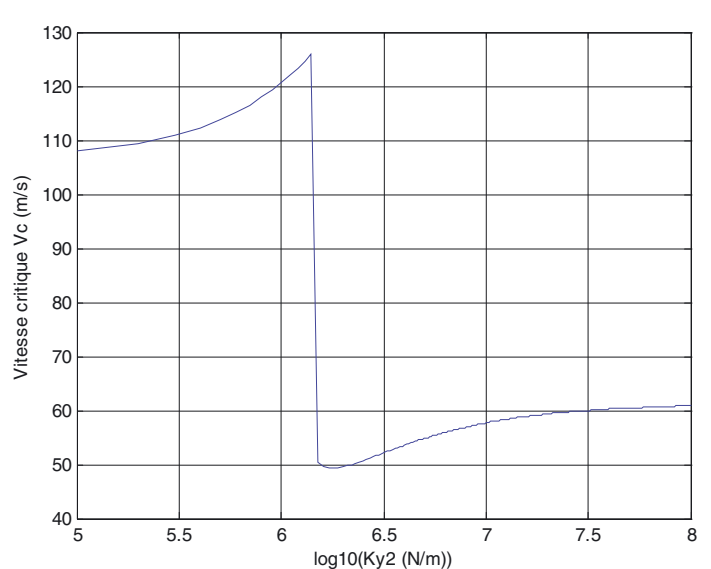

Fig. 6. Variation de $V_{\text {c }}$ en fonction de $\bar{K}_{y}$.

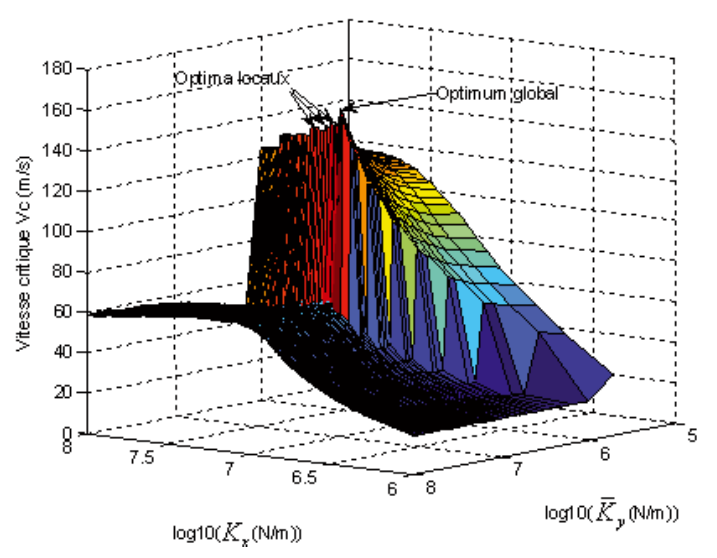

Fig. 7. Variation de $V_{\mathrm{c}}$ en fonction de $K_{x}$ et $K_{y}$.

l'une des deux rigidités engendre une diminution brusque de la vitesse critique. Ce phénomène correspond à une bifurcation de la solution [2] : à deux valeurs très voisines de la rigidité correspondent deux valeurs de la vitesse critique. La figure 7 montre la forte discontinuité de la variation de la vitesse critique. Cette fonction présente un optimum global et plusieurs optima locaux.

Les paramètres d'inertie qui influent le plus sur la vitesse critique sont les masses de la caisse $M_{\mathrm{c}}$ et du châssis de bogie $M_{\mathrm{b}}$. Pour les paramètres géométriques, ce sont la conicité équivalente $\gamma_{\mathrm{e}}$ et l'empattement des essieux $2 a$ qui ont une influence considérable sur la variation de $V_{\mathrm{c}}$ (Fig. 8).

L'augmentation de la masse du bogie engendre une diminution de la vitesse critique. En effet, les forces d'inertie de la masse oscillante favorisent l'instabilité. La conicité équivalente est le paramètre qui définit la géométrie de contact entre le rail et la roue. L'augmentation de ce paramètre engendre une diminution de la vitesse critique. Ce résultat théorique est en parfait accord avec l'expérience. D'ailleurs, ce paramètre augmente au cours du temps à cause de l'usure des roues et des rails. Alors, on a intérêt à réduire le glissement entre les roues et les rails, pour ralentir l'augmentation de la conicité équivalente. Cette étude a montré que plusieurs paramètres de construction du 


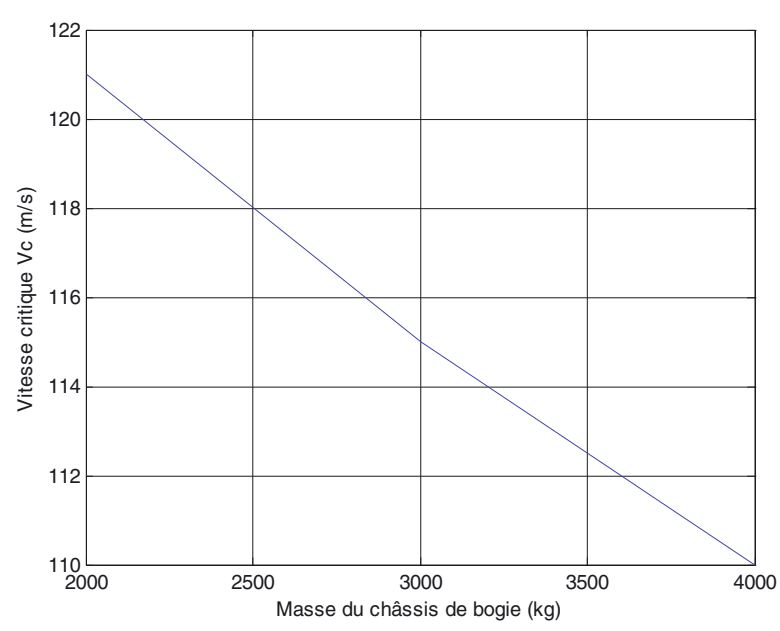

(a)

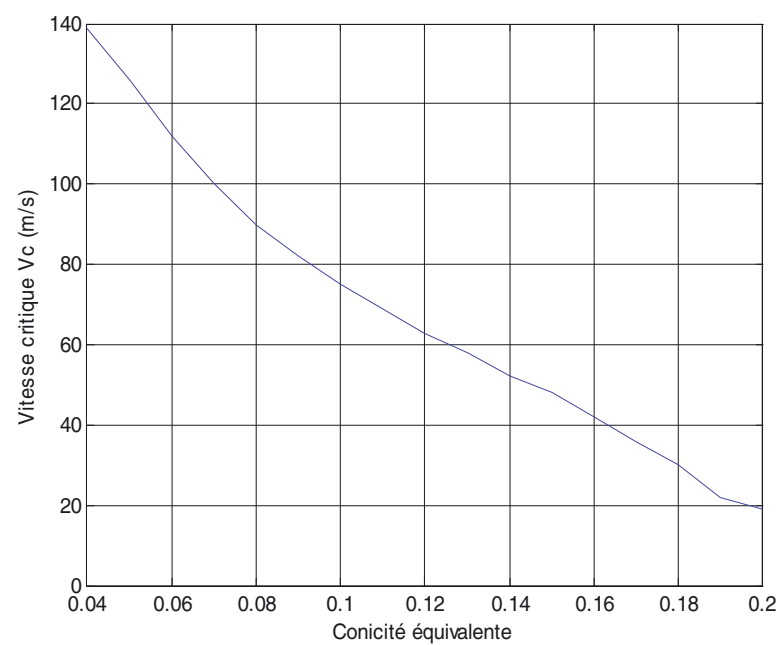

(b)

Fig. 8. (a) Variation de $V_{\mathrm{c}}$ en fonction de $M_{\mathrm{b}}$, (b) variation de $V_{\text {c }}$ en fonction de $\gamma_{\mathrm{e}}$.

véhicule influent sur la stabilité transversale du véhicule; ainsi, pour optimiser cette stabilité, on cherche les valeurs optimales de ces paramètres. L'expression de la vitesse critique, en fonction des paramètres à optimiser, est inconnue. Pour cela, la méthode d'optimisation choisie et qui convient bien à ce cas, est la méthode des algorithmes génétiques. En effet, He et McPhee [2] ont montré aussi que cette méthode est plus efficace que l'algorithme de Simplex dont la fiabilité diminue lorsque le nombre de variables augmente.

\subsection{Optimisation de la stabilité transversale par l'AG}

\subsubsection{Formulation du problème}

Les paramètres de construction à optimiser sont ceux qui influent considérablement sur la variation de la vitesse critique, donc sur la stabilité transversale du véhicule. Ces
Tableau 1. Solutions optimales.

\begin{tabular}{|l|l|}
\hline$K_{x}\left(\mathrm{~N} \cdot \mathrm{m}^{-1}\right)$ & $5 \times 10^{7}$ \\
\hline$K_{y}\left(\mathrm{~N} \cdot \mathrm{m}^{-1}\right)$ & $1,3 \times 10^{6}$ \\
\hline $\bar{K}_{x}\left(\mathrm{~N} \cdot \mathrm{m}^{-1}\right)$ & $10^{6}$ \\
\hline $\bar{K}_{y}\left(\mathrm{~N} \cdot \mathrm{m}^{-1}\right)$ & $10^{6}$ \\
\hline$\gamma_{\mathrm{e}}$ & 0,05 \\
\hline$a(\mathrm{~m})$ & 2 \\
\hline$M_{c}(\mathrm{~kg})$ & 45000 \\
\hline$M_{b}(\mathrm{~kg})$ & 3000 \\
\hline$V_{\mathrm{c}}\left(\mathrm{m} \cdot \mathrm{s}^{-1}\right)$ & 256 \\
\hline
\end{tabular}

paramètres sont ceux cités précédemment dans la partie précédente. Ainsi, le problème est formulé comme suit :

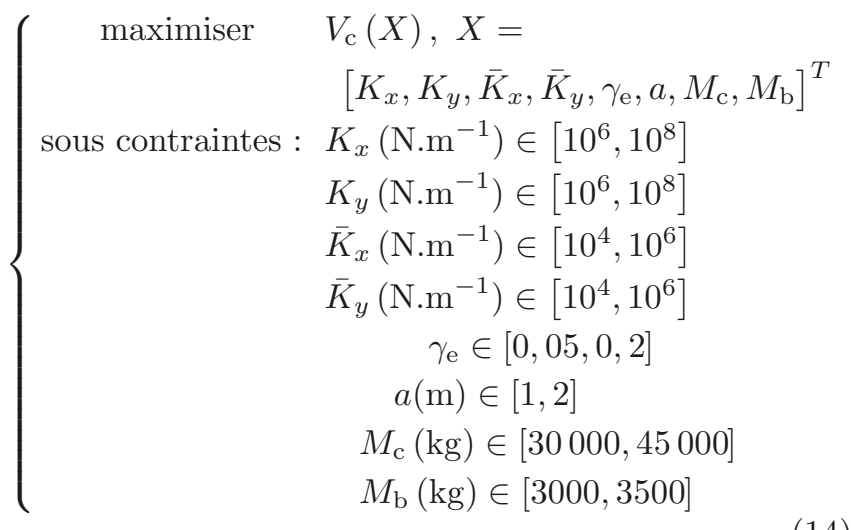

Les intervalles d'existence des variables (l'espace de recherche) sont choisis tels qu'ils contiennent les valeurs nominales des paramètres à optimiser. Les rigidités de la suspension secondaire sont plus souples pour des raisons de confort et pour éviter les valeurs correspondant à une bifurcation de la solution.

La population est de 30 individus choisis aléatoirement dans l'espace de recherche. L'exécution s'arrête quand l'algorithme accomplit un nombre maximum de générations $G_{\max }=80$.

\subsubsection{Résultats d'optimisation}

Après l'exécution de l'algorithme, on obtient les solutions optimales et la valeur de la vitesse critique correspondante qui sont illustrées dans le tableau 1.

Puisque les valeurs optimales des rigidités de la suspension secondaire correspondent aux bornes supérieures de leurs intervalles d'existence, ces valeurs ne correspondent pas au phénomène de bifurcation. La figure 9 montre l'évolution de l'aptitude $\left(V_{\mathrm{c}}\right)$ du meilleur individu en fonction des générations.

\section{Optimisation du comportement en courbe du véhicule}

Dans cette section, on présente le modèle du véhicule et la méthode d'étude de son comportement en courbe. 


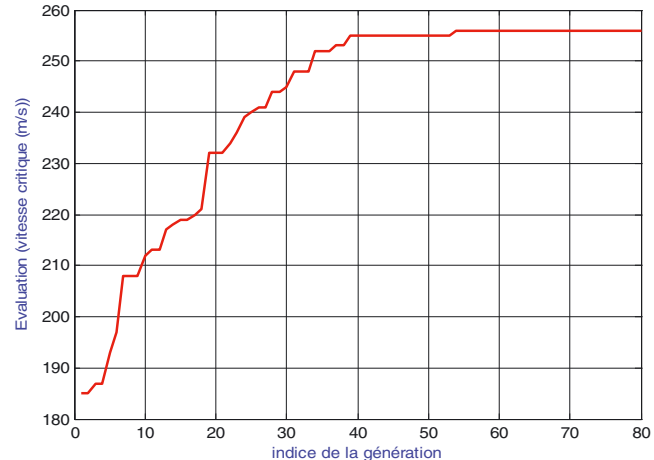

Fig. 9. Évolution de l'aptitude du meilleur individu.

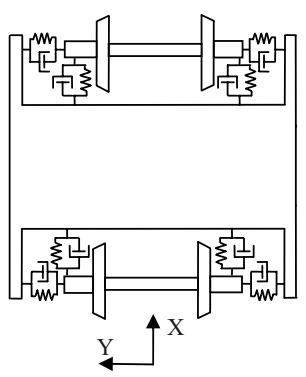

Fig. 10. Modèle du véhicule pour la simulation en courbe.

Les résultats de cette étude montrent l'influence des paramètres de construction du véhicule sur son comportement en courbe. Après, on applique la méthode des algorithmes génétiques afin d'optimiser le comportement en courbe de ce véhicule.

\subsection{Modèle du véhicule}

Le modèle étudié est présenté par la figure 10.

Les paramètres de la dynamique transversale des différents solides constituant le véhicule sont les mêmes utilisés pour l'étude de sa stabilité transversale.

\subsection{Circulation en pleine courbe}

Dans une voie en courbe de rayon constant, un essieu en circulation libre sur la voie, dans un mouvement de roulement sans glissement, prend une position stable. Cette position est caractérisée par un déplacement transversal $[12] y_{0}$ tel que :

$$
y_{0}=\frac{e_{0} r_{0}}{\gamma_{\mathrm{e}} R_{\mathrm{c}}}
$$

Elle sera prise comme origine des déplacements transversaux.

On pose :

$$
\bar{Y}=\frac{\bar{Y}_{1}+\bar{Y}_{2}}{2} \quad \text { et } \quad \bar{\alpha}=\frac{\bar{Y}_{1}-\bar{Y}_{2}}{2 \bar{A}}
$$

$$
\begin{aligned}
\bar{Y}_{1} & =\bar{Y}_{1}^{*}+Y_{1}^{*}+\frac{y_{11}^{*}+y_{12}^{*}}{2}+y_{0} \\
Y_{1} & =Y_{1}^{*}+\frac{y_{11}^{*}+y_{12}^{*}}{2}+y_{0} \\
\alpha_{k} & =\alpha_{k}^{*}+\frac{y_{k 1}^{*}-y_{k 2}^{*}}{2 a} \\
y_{k i} & =y_{k i}^{*}+y_{0}
\end{aligned}
$$

En pleine courbe, le dévers de la voie est supposé constant, ainsi que son rayon de courbure $\left(R_{\mathrm{c}}\right)$. Le véhicule est supposé circuler en régime stable (vitesse de circulation constante légèrement supérieure à la vitesse d'équilibre). Ainsi, il est possible de négliger :

- les forces d'inertie et d'amortissement devant les forces de rappel élastiques;

- les mouvements de roulis des différents solides.

En écrivant le modèle dynamique du véhicule en circulation, sur une voie en courbe de rayon constant, les hypothèses simplificatrices citées ci-dessus et le changement de variables ont permis de découpler le système mécanique (caisse, deux bogies et quatre essieux) et de pouvoir étudier le comportement du véhicule en courbe en se référant à un seul bogie avec deux essieux [12]. On considère le premier bogie dans le sens de la marche, les degrés de liberté sont : $\alpha_{1}^{*}, y_{11}^{*}, y_{12}^{*}, \alpha_{11}$ et $\alpha_{12}$. On suppose aussi que la charge supportée par les roues gauches est égale à celle supportée par les roues droites.

\subsection{Les forces de pseudo-glissement}

Dans le cas de la circulation du véhicule en courbe de grand rayon $\left(R_{\mathrm{c}} \geq 500 \mathrm{~m}\right)$, les expressions des forces de pseudo-glissement s'obtiennent par la théorie linéaire de Kalker. Pour une circulation en courbe de faible rayon (100 $\left.\mathrm{m} \leq R_{\mathrm{c}}<500 \mathrm{~m}\right)$, les glissements réduits deviennent plus importants. Dans ce cas, on utilise la loi de saturation proposée par Johnson et Vermeulen, basée sur les coefficients de Kalker [1]. Les expressions analytiques des forces de pseudo-glissement deviennent :

$$
\begin{aligned}
X_{k i 1}= & -C_{11}\left(-\frac{\gamma_{\mathrm{e}}}{r_{0}} y_{k i}+\frac{e_{0}}{R_{\mathrm{c}}}\right)\left\{1-\frac{C_{11}}{3 \mu N}\left(-\frac{\gamma_{\mathrm{e}}}{r_{0}} y_{k i}+\frac{e_{0}}{R_{\mathrm{c}}}\right)\right. \\
& \left.+\frac{C_{11}^{2}}{27 \mu^{2} N^{2}}\left(-\frac{\gamma_{\mathrm{e}}}{r_{0}} y_{k i}+\frac{e_{0}}{R_{\mathrm{c}}}\right)^{2}\right\} \\
X_{k i 2}= & -C_{11}\left(\frac{\gamma_{\mathrm{e}}}{r_{0}} y_{k i}-\frac{e_{0}}{R_{\mathrm{c}}}\right)\left\{1-\frac{C_{11}}{3 \mu N}\left(\frac{\gamma_{\mathrm{e}}}{r_{0}} y_{k i}-\frac{e_{0}}{R_{\mathrm{c}}}\right)\right. \\
& \left.+\frac{C_{11}^{2}}{27 \mu^{2} N^{2}}\left(\frac{\gamma_{\mathrm{e}}}{r_{0}} y_{k i}-\frac{e_{0}}{R_{\mathrm{c}}}\right)^{2}\right\} \\
T_{k i 1}= & C_{22} \alpha_{k i}\left\{1+\frac{C_{22}}{3 \mu N} \alpha_{k i}+\frac{C_{22}^{2}}{27 \mu^{2} N^{2}} \alpha_{k i}^{2}\right\} \\
T_{k i 2}= & C_{22} \alpha_{k i}\left\{1+\frac{C_{22}}{3 \mu N} \alpha_{k i}+\frac{C_{22}^{2}}{27 \mu^{2} N^{2}} \alpha_{k i}^{2}\right\}
\end{aligned}
$$




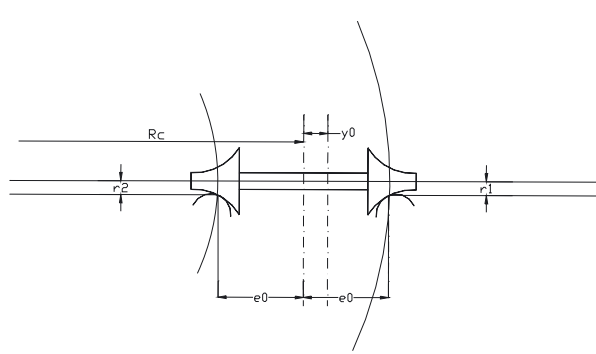

Fig. 11. Essieu en mouvement libre sur une voie en courbe.

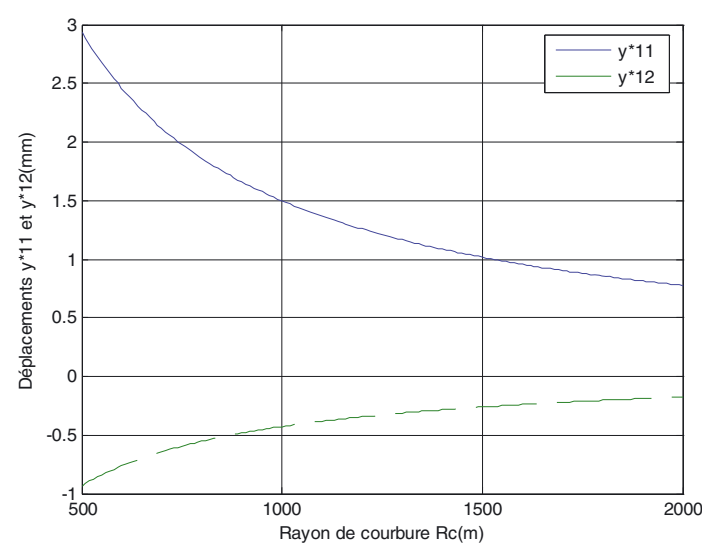

Fig. 12. Influence de $R_{\mathrm{c}}$.

\subsection{Résolution du système}

\subsubsection{Système linéaire}

Pour les grands rayons de courbure, le système linéaire obtenu est de la forme suivante :

$$
\mathrm{A}\{q\}=\{B\}
$$

avec $:\{q\}=\left[\alpha_{1}^{*}, y_{11}^{*}, y_{12}^{*}, \alpha_{11}, \alpha_{12}\right]^{T}$.

Par suite, on obtient le vecteur $\{q\}$ comme suit :

$$
\{q\}=\mathrm{A}^{-1}\{B\}
$$

Pour les résultats, on s'intéresse à l'influence de la variation des paramètres de construction du véhicule sur la variation des déplacements transversaux et $y_{12}^{*}$. Au début, on a analysé l'influence de la variation du rayon de courbure sur les déplacements (Fig. 12).

On remarque que le déplacement transversal diminue lorsque le rayon de courbure augmente. En effet, l'augmentation de ce dernier engendre une diminution de la force centrifuge appliquée sur le véhicule.

Par suite, on garde le rayon de courbure constant $R_{\mathrm{c}}=500 \mathrm{~m}$. La figure 13 montre l'influence de la variation des rigidités longitudinale et transversale de la suspension primaire $K_{x}$ et $K_{y}$.

L'augmentation de $K_{y}$ n'engendre une augmentation du déplacement qu'à partir d'une valeur de $2,5 \times$ $10^{6} \mathrm{~N} . \mathrm{m}^{-1}$ de $K_{x}$. En effet, l'augmentation des rigidités des ressorts rend le système mécanique (châssis de bogie, essieux) rigide et ainsi les efforts élastiques sont transmis

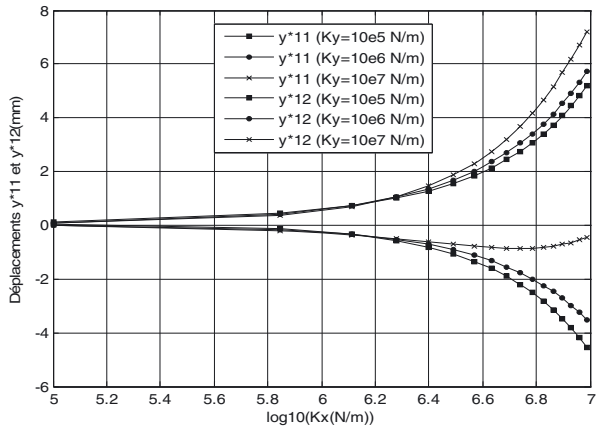

Fig. 13. Influence de la variation de $K_{x}$ et $K_{y}$.

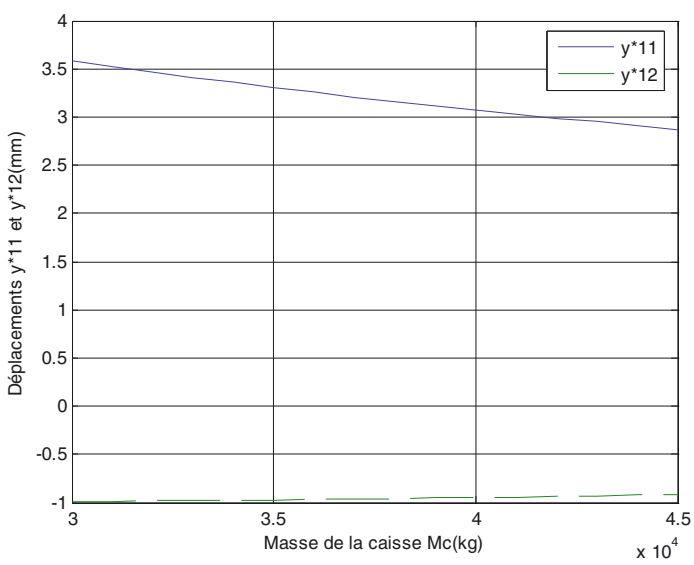

(a)

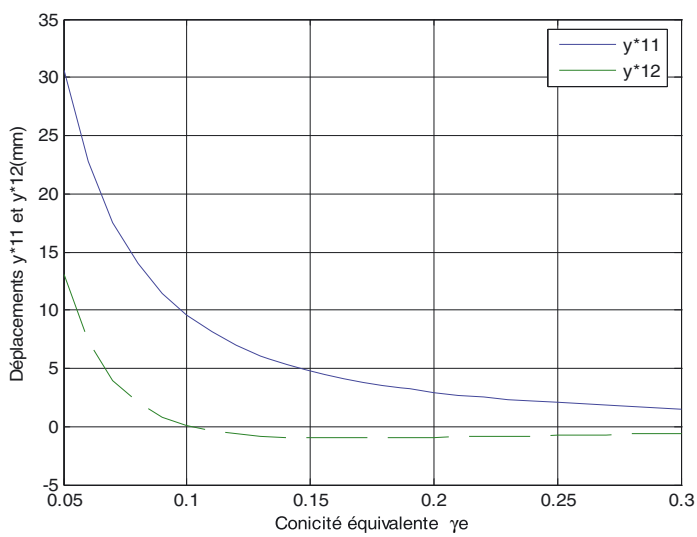

(b)

Fig. 14. (a) Influence de $M_{\mathrm{c}}$, (b) influence de $\gamma_{\mathrm{e}}$.

directement aux essieux. $y_{11}^{*}$ est toujours positif et $y_{12}^{*}$ est négatif : l'essieu avant se déplace vers l'extérieur de la courbe et l'essieu arrière prend une position centrée dans la voie. Les paramètres qui influent sur le déplacement transversal sont les masses de la caisse $M_{\mathrm{c}}$ et du châssis de bogie $M_{\mathrm{b}}$. Pour les paramètres géométriques, ce sont la conicité équivalente $\gamma_{\mathrm{e}}$ et l'empattement des essieux $2 a$ qui ont une influence considérable sur le déplacement transversal (Fig. 14). 


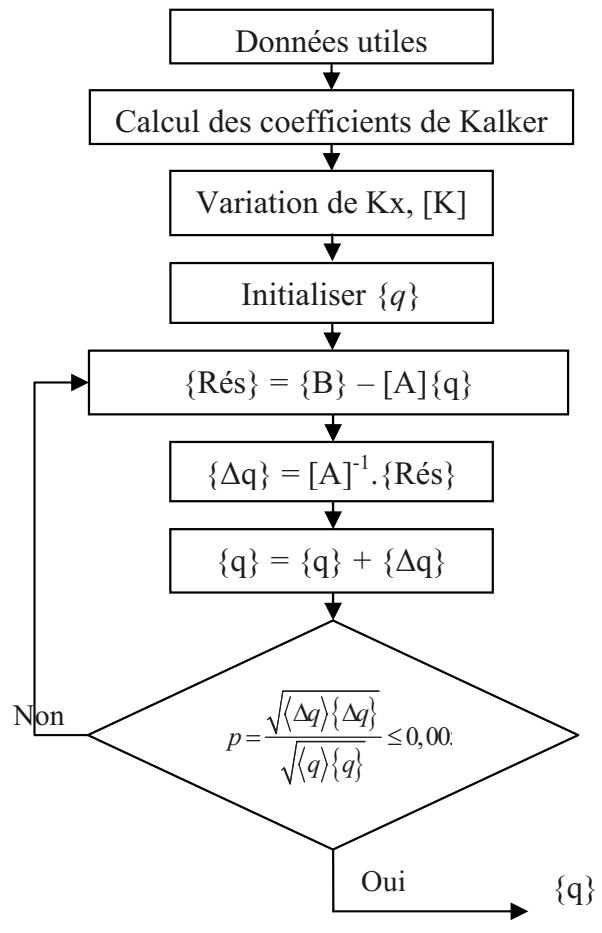

Fig. 15. Algorithme de calcul de $\{q\}$ en fonction de $K_{x}$.

L'augmentation des masses de la caisse et du châssis de bogie engendre une diminution des déplacements transversaux. En effet, l'augmentation de ces deux masses entraîne une augmentation de la charge normale exercée sur les rails, ce qui diminue les déplacements transversaux des essieux. On remarque que l'augmentation de $\gamma_{\mathrm{e}}$ entraîne une diminution des déplacements transversaux des essieux. Effectivement, l'augmentation du rayon de courbure du profil de la roue engendre une augmentation de l'adhérence entre le rail et la roue. Par contre, l'augmentation de l'empattement entraîne une augmentation des déplacements transversaux. En effet, l'augmentation de a engendre une élévation de la rigidité de la liaison élastique, ce qui facilite la transmission des efforts aux essieux.

\subsubsection{Système non-linéaire}

Pour les faibles rayons de courbure, les expressions analytiques des forces de pseudo-glissement sont calculées à partir de la méthode de Johnson et Vermeulen [1] . Les équations de mouvement d'un bogie et deux essieux forment un système non-linéaire de la forme suivante :

$$
[A(q)]\{q\}=\{B\}
$$

avec : $\{q\}=\left[\alpha_{1}^{*}, y_{11}^{*}, y_{12}^{*}, \alpha_{11}, \alpha_{12}\right]^{T}$.

La méthode utilisée pour résoudre ce système est la méthode de substitution : méthode itérative qui consiste à chercher un vecteur $\{q\}$ qui rend le résidu $[A(q)]\{q\}-\{B\}$ aussi proche que possible de 0 . L'algorithme présenté par la figure 15 montre la manière de calcul du vecteur $\{q\}$ en utilisant cette méthode.

\subsection{Optimisation du comportement en courbe par I'AG}

\subsubsection{Formulation du problème}

L'objectif de cette partie est de trouver les valeurs optimales des paramètres de construction du véhicule qui améliorent son comportement en courbe; ceci pour éviter le contact des boudins des roues avec les rails et les glissements entre la roue et le rail afin d'éliminer les risques de déraillement et réduire les usures des roues et des rails. Ainsi, on doit minimiser les déplacements transversaux des essieux. Les figures précédentes illustrent l'effet de la variation des paramètres de construction du véhicule sur les déplacements transversaux, et montrent que le déplacement de l'essieu mené $y_{12}^{*}$ est toujours inférieur aux déplacements de l'essieu directeur $y_{11}^{*}$. Alors, il suffit de trouver les valeurs optimales des paramètres de construction qui minimisent $y_{11}^{*}$; ces valeurs minimisent aussi $y_{12}^{*}$. Aussi, on a remarqué que le déplacement transversal diminue lorsque le rayon de courbure augmente. Alors, l'optimisation doit être faite pour le rayon de courbure le plus faible. Ainsi, pour la circulation en courbe, le problème est formulé comme suit :

$$
\left\{\begin{aligned}
\text { minimiser } y_{11}^{*}(X), & X= \\
\text { sous } \quad & {\left[K_{x}, K_{y}, \bar{K}_{x}, \gamma_{e}, a, M_{c}, M_{b}\right]^{T} } \\
\text { contraintes }: K_{x}\left(\mathrm{~N}_{y}\left(\mathrm{~N}^{-1}\right)\right. & \in\left[10^{6}, 10^{8}\right] \\
\bar{K}_{x}\left(\mathrm{~N}^{-1}\right) & \in\left[10^{6}, 10^{8}\right] \\
\gamma_{\mathrm{e}} & \in\left[10^{4}, 10^{6}\right] \\
a(\mathrm{~m}) & \in[1,2] \\
M_{\mathrm{c}}(\mathrm{kg}) & \in[30000,0], 45000] \\
M_{\mathrm{b}}(\mathrm{kg}) & \in[3000,3500]
\end{aligned}\right.
$$

La population est de 20 individus choisis aléatoirement dans l'espace de recherche. L'exécution s'arrête quand l'algorithme accomplit un nombre maximum de générations $G_{\max }=40$.

\subsubsection{Résultats}

Après l'exécution de l'algorithme, on obtient les solutions optimales à partir de deux systèmes d'équations. Le premier système d'équations linéaires est utilisé pour les calculs d'optimisation en courbe pour les rayons de courbure $R_{\mathrm{c}}$ supérieurs à $500 \mathrm{~m}$; alors que le système d'équations non-linéaires est utilisé pour les rayons de courbure $R_{\mathrm{c}}$ inférieurs à $500 \mathrm{~m}$.

\subsubsection{Système linéaire}

Pour les rayons de courbure $R_{\mathrm{c}}$ supérieurs à $500 \mathrm{~m}$, les résultats sont illustrés dans le tableau 2 .

La figure 16 montre l'évolution de l'aptitude $\left(y_{11}^{*}\right)$ du meilleur individu en fonction des générations. 


$$
F R=\sqrt{\left(C_{11} \frac{\gamma_{\mathrm{e}}}{r_{0}} y_{11}^{*}\right)^{2}+\left(C_{22} \alpha_{11}+C_{23} \frac{\gamma_{\mathrm{e}}}{R_{1} \gamma_{0} r_{0}} y_{11}^{*}+C_{23} \frac{\gamma_{\mathrm{e}}}{R_{1} \gamma_{0} r_{0}} y_{0}+\frac{C_{23}}{R_{c}}+\frac{C_{23} \gamma_{0}}{r_{0}}\right)^{2}}
$$

Tableau 2. Solutions optimales.

\begin{tabular}{|l|l|}
\hline$K_{x}\left(\mathrm{~N} \cdot \mathrm{m}^{-1}\right)$ & $10^{6}$ \\
\hline$K_{y}\left(\mathrm{~N} \cdot \mathrm{m}^{-1}\right)$ & $10^{8}$ \\
\hline $\bar{K}_{x}\left(\mathrm{~N} \cdot \mathrm{m}^{-1}\right)$ & $10^{4}$ \\
\hline$\gamma_{\mathrm{e}}$ & 0,2 \\
\hline$a(\mathrm{~m})$ & 1 \\
\hline$M_{c}(\mathrm{~kg})$ & 45000 \\
\hline$M_{b}(\mathrm{~kg})$ & 3500 \\
\hline$y_{11}(\mathrm{~mm})$ & 3,69 \\
\hline
\end{tabular}

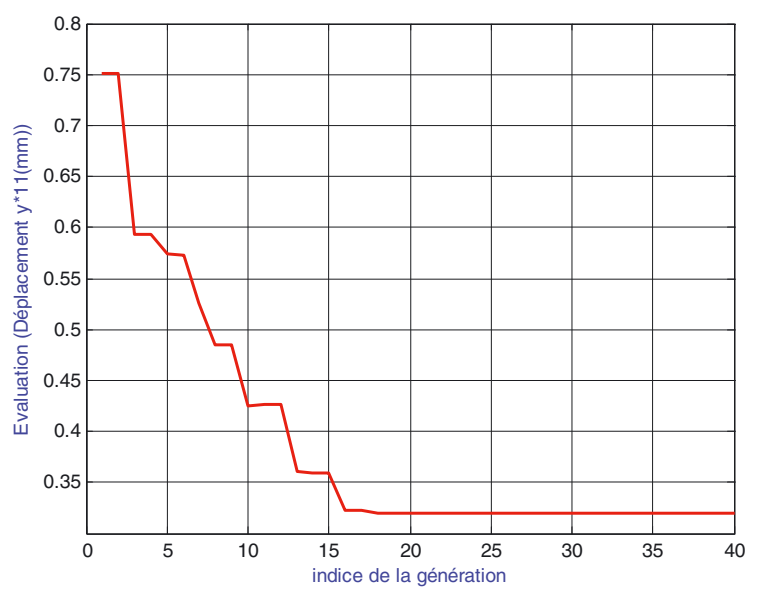

Fig. 16. Évolution de l'aptitude du meilleur individu.

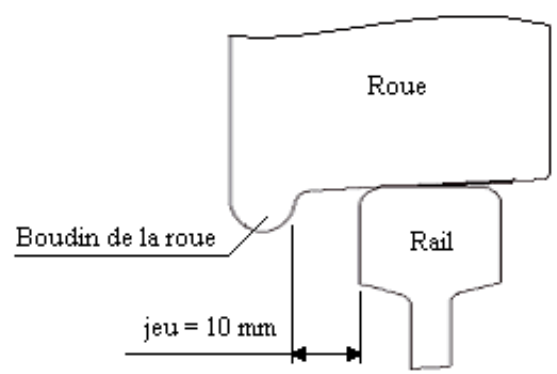

Fig. 17. Jeu entre le boudin de la roue et le rail.

Pour ces valeurs optimales, on a :

$$
\begin{aligned}
& y_{0}=3,37 \mathrm{~mm} \\
& \Rightarrow y_{1}=y_{0}+y_{11}^{*}=3,69 \mathrm{~mm}
\end{aligned}
$$

Le déplacement total de l'essieu ne dépasse pas le jeu entre le boudin de la roue et le rail (Fig. 17) et le contact boudin-rail est évité.

De plus, pour éviter le glissement, il faut vérifier que le module de la résultante des forces de pseudo-glissement
Tableau 3. Solution optimale.

\begin{tabular}{|l|l|}
\hline$K_{x}\left(\mathrm{~N} \cdot \mathrm{m}^{-1}\right)$ & $10^{6}$ \\
\hline$K_{y}\left(\mathrm{~N} \cdot \mathrm{m}^{-1}\right)$ & $10^{8}$ \\
\hline $\bar{K}_{x}\left(\mathrm{~N} \cdot \mathrm{m}^{-1}\right)$ & $10^{4}$ \\
\hline$\gamma_{\mathrm{e}}$ & 0,2 \\
\hline$a(\mathrm{~m})$ & 1 \\
\hline$M_{\mathrm{c}}(\mathrm{kg})$ & 45000 \\
\hline$M_{\mathrm{b}}(\mathrm{kg})$ & 3500 \\
\hline$y_{11}^{*}(\mathrm{~mm})$ & 1,2 \\
\hline
\end{tabular}

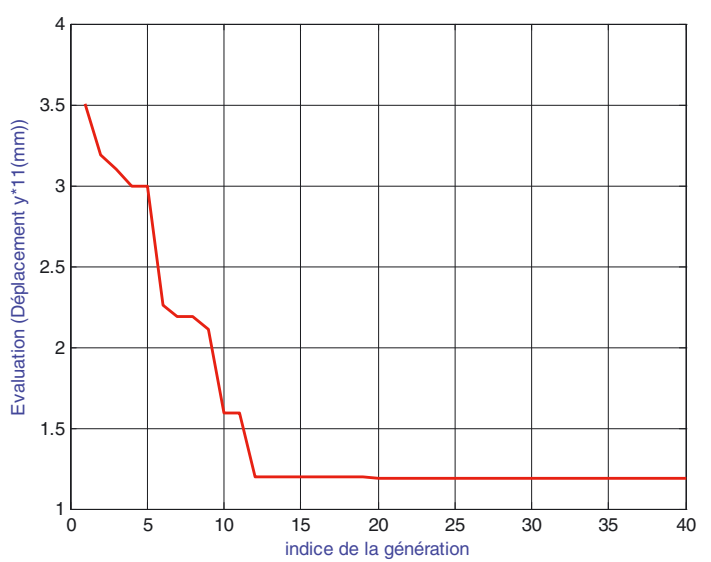

Fig. 18. Évolution de l'aptitude du meilleur individu.

$(F R)$ est inférieur à l'amplitude de la force de glissement donnée par la loi de Coulomb :

$$
F R<\mu N
$$

avec :

$$
\text { voir équation ci-dessus }
$$

$\mu$ : coefficient de frottement, $N$ : charge normale par roue;

On a $F R=1,02 \times 10^{4} N<\mu N=1,5 \times 10^{4} N$, donc pas de glissement.

\subsubsection{Système non-linéaire}

Pour le système non-linéaire et pour un rayon de courbure $R_{\mathrm{c}}=100 \mathrm{~m}$, les résultats sont illustrés dans le tableau 3 .

La figure 18 montre l'évolution de l'aptitude $\left(y_{11}^{*}\right)$ du meilleur individu en fonction des générations.

Pour le rayon de courbure de $100 \mathrm{~m}$, la quantité $y_{0}$, qui est égale à $16,8 \mathrm{~mm}$, dépasse le jeu entre le boudin de la roue et le rail. Dans ce cas, le boudin de la roue touche le rail et exerce sur ce dernier une force de poussée $F_{k i}$ de même sens que le déplacement (Fig. 19). Ainsi, 


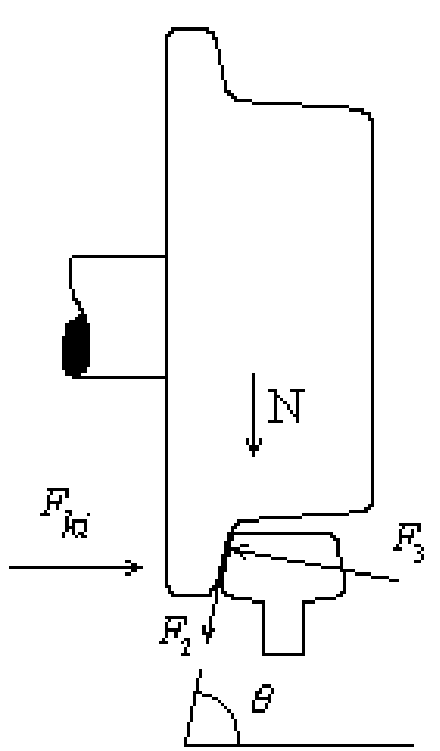

Fig. 19. Contact boudin-rail.

cette force remplace $y_{k i}^{*}$ parmi les inconnues du système à résoudre. Les termes en $y_{k i}^{*}$, déjà connus, passent au second membre.

Au contact boudin-rail, deux réactions sont appliquées par le rail sur la roue : la force normale $F_{3}$ et la force de pseudo-glissement latérale $F_{2}$. Le plan de contact fait un angle $\theta$ avec l'horizontale. L'équilibre des forces dans les deux directions latérale et verticale au point de contact mène à l'équation suivante :

$$
\frac{F_{k i}}{N}=\frac{\operatorname{tg} \theta-F_{2} / F_{3}}{1+F_{2} \operatorname{tg} \theta / F_{3}}
$$

Cette équation donne le taux $F_{k i} / N$ minimal pour lequel le déraillement peut être produit pour n'importe quelle valeur de $F_{2} / F_{3}$ à un angle de contact maximal $\theta$ [15]. Ce taux diminue lorsque $F_{2} / F_{3}$ augmente. La valeur maximale du rapport $F_{2} / F_{3}$ est égale au coefficient de frottement $\mu$. Ainsi, pour éviter le déraillement, il faut que :

$$
\frac{F_{k i}}{N}<\frac{\operatorname{tg} \theta_{\max }-\mu}{1+\mu \operatorname{tg} \theta_{\max }}
$$

La valeur de la force de poussée $F_{11}$ correspondant à $y_{11}^{*}$ minimal trouvé précédemment est :

$$
F_{11}=3,37 \times 10^{4} \mathrm{~N}
$$

L'angle de contact $\theta_{\text {max }}$ est égal à 75 degrés [15]. Alors, pour cette valeur, on a :

$$
\frac{F_{11}}{N}=0,38<\frac{\operatorname{tg} \theta_{\max }-\mu}{1+\mu \operatorname{tg} \theta_{\max }}=1,06
$$

Ainsi, pour un rayon de courbure de $100 \mathrm{~m}$, on ne peut pas éviter le contact entre le boudin de la roue et le rail, mais ceci se fait sans déraillement du véhicule. C'est le cas aussi pour un rayon de $150 \mathrm{~m}$. Pour un rayon de courbure de $200 \mathrm{~m}$, le véhicule peut circuler sans
Tableau 4. Valeurs optimales des paramètres de construction du véhicule.

\begin{tabular}{|l|l|l|}
\cline { 2 - 3 } \multicolumn{1}{c|}{} & $\begin{array}{l}\text { Stabilité } \\
\text { transversale }\end{array}$ & $\begin{array}{l}\text { Comportement } \\
\text { en courbe }\end{array}$ \\
\hline$K_{x}\left(\mathrm{~N} \cdot \mathrm{m}^{-1}\right)$ & $5 \times 10^{7}$ & $10^{6}$ \\
\hline$K_{y}\left(\mathrm{~N} \cdot \mathrm{m}^{-1}\right)$ & $1,3 \times 10^{6}$ & $10^{8}$ \\
\hline $\bar{K}_{x}\left(\mathrm{~N} \cdot \mathrm{m}^{-1}\right)$ & $10^{6}$ & $10^{4}$ \\
\hline$\gamma_{\mathrm{e}}$ & 0,05 & 0,2 \\
\hline$a(\mathrm{~m})$ & 2 & 1 \\
\hline$M_{\mathrm{c}}(\mathrm{kg})$ & 45000 & 45000 \\
\hline$M_{\mathrm{b}}(\mathrm{kg})$ & 3000 & 3500 \\
\hline
\end{tabular}

contact boudin-rail et sans glissement. En effet, pour les mêmes valeurs des paramètres de construction du véhicule trouvées précédemment, on a :

$y_{0}=8,4 \mathrm{~mm}$

$\Rightarrow y_{1}=y_{0}+y_{11}^{*}=9 \mathrm{~mm}$

et $F R=1,74 \times 10^{4} N<\mu N=2,8 \times 10^{4} N$

\section{Conclusion}

La majorité des valeurs optimales des paramètres de construction qui donnent un bon comportement en courbe du véhicule sont en contradiction avec celles trouvées pour l'optimisation de la stabilité transversale en alignement (Tab. 4).

Il y a incompatibilité entre une bonne stabilité transversale en alignement et un bon comportement en courbe du véhicule. En effet, les valeurs des paramètres de construction nécessaires pour avoir une vitesse critique élevée en alignement entraînent, en circulation en courbe, un contact rapide des roues avec les rails, des glissements roue-rail provoquant une usure rapide des roues et des efforts d'interaction élevés pouvant provoquer le déraillement du véhicule. D'où, la nécessité de trouver les valeurs optimales des paramètres de construction qui permettent à la fois d'avoir une bonne stabilité transversale du véhicule, circulant en alignement, et un bon comportement en courbe. Pour ce faire, l'utilisation des algorithmes génétiques convient bien à ce cas.

\subsection{Optimisations multi-objectives des paramètres de construction du véhicule}

Dans cette partie, on appliquera la méthode des algorithmes génétiques pour faire une optimisation multiobjective. Le but est de trouver les valeurs optimales des paramètres de construction du véhicule permettant une bonne stabilité transversale lors d'une circulation en alignement et un bon comportement en courbe.

\subsubsection{Formulation du problème}

L'objectif de notre problème est de maximiser la vitesse critique lorsque le véhicule circule en alignement et 
minimiser le déplacement transversal des essieux lors de sa circulation en courbe. Ainsi, on a un problème à deux fonctions objectives qui peut être formulé comme suit :

$$
\left\{\begin{array}{cl}
\text { maximiser } & V_{\mathrm{c}}(X), X= \\
& {\left[K_{x}, K_{y}, \bar{K}_{x}, \gamma_{\mathrm{e}}, a, M_{\mathrm{c}}, M_{\mathrm{b}}\right]^{T}} \\
& y_{11}^{*}(X) \\
\text { et minimiser } & K_{x}\left(\mathrm{~N} \cdot \mathrm{m}^{-1}\right) \in\left[10^{6}, 10^{8}\right] \\
& K_{y}\left(\mathrm{~N} \cdot \mathrm{m}^{-1}\right) \in\left[10^{6}, 10^{8}\right] \\
& \bar{K}_{x}\left(\mathrm{~N} \cdot \mathrm{m}^{-1}\right) \in\left[10^{4}, 10^{6}\right] \\
& \gamma_{\mathrm{e}} \in[0,05,0,2] \\
& a(\mathrm{~m}) \in[1,2] \\
& M_{\mathrm{c}}(\mathrm{kg}) \in[30000,45000] \\
& M_{\mathrm{b}}(\mathrm{kg}) \in[3000,3500] \\
& y_{11}^{*}<10-y_{0}
\end{array}\right.
$$

Puisque $y_{11}^{*}$ ne dépend pas de la rigidité transversale de la suspension secondaire, la valeur de $\bar{K}_{y}$ adoptée est égale à sa valeur optimale trouvée lors de l'optimisation de la stabilité transversale du véhicule circulant en alignement. La contrainte sur le déplacement $y_{11}^{*}$ est satisfaite si les solutions obtenues permettent d'éviter le contact entre le boudin de la roue et le rail.

\subsubsection{Algorithme d'optimisation}

La méthode d'optimisation utilisée est basée sur le principe de dominance de Pareto. De plus, ce principe est utilisé pour satisfaire la contrainte sur $y_{11}^{*}$ (système (27)). Les solutions ne satisfaisant pas cette contrainte sont dominées par celles qui la satisfont.

\subsubsection{Initialisation}

La population est de 100 individus choisis aléatoirement dans l'espace de recherche. L'exécution s'arrête quand l'algorithme accomplit un nombre maximum de générations $G_{\max }=200$.

\subsubsection{2. Évaluation}

Pour chaque individu, on calcule la vitesse critique et le déplacement transversal correspondant. Ensuite, on assigne à chaque individu une valeur d'aptitude selon la méthode proposée par Goldberg [4].

\subsubsection{Préservation de la diversité}

Pour préserver la diversité pendant la recherche des solutions optimales, on a utilisé la méthode de partage des ressources [9]. Cette méthode consiste à donner une nouvelle aptitude partagée $s_{k}$ à chaque individu comme suit :

$$
s_{k}=\frac{f_{k}}{\sum_{j=1}^{n} \operatorname{sh}(d(X, Y))}
$$

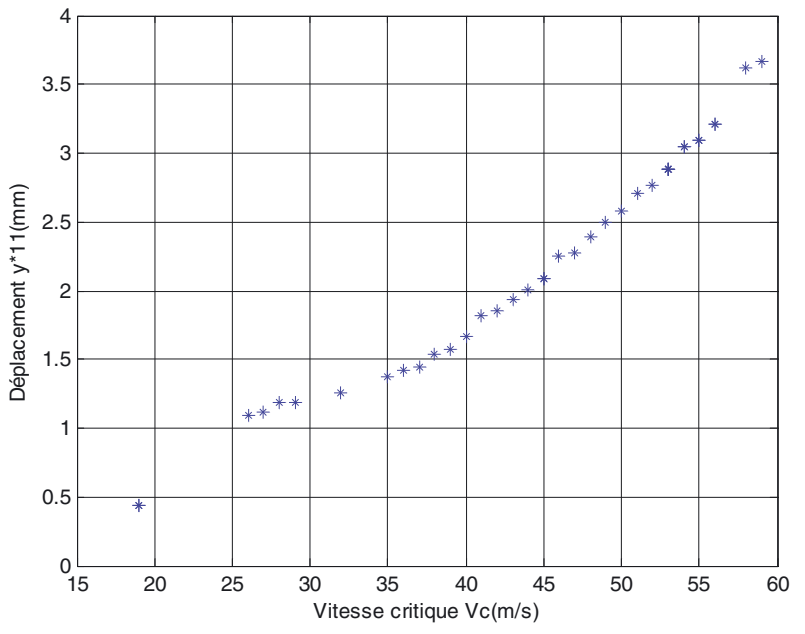

Fig. 20. Solutions optimales $\left(R_{c}=500 \mathrm{~m}\right)$.

avec $f_{k}:$ l'ancienne aptitude

$$
\operatorname{sh}(d(X, Y))= \begin{cases}1-\left(\frac{d(X, Y)}{\sigma_{\text {share }}}\right)^{\alpha} & \text { si } d(X, Y)<\sigma_{\text {share }} \\ 0 & \text { sinon }\end{cases}
$$

et $d(X, Y)=\left(\sum_{i=1}^{m}\left|x_{i}-y_{i}\right|^{2}\right)^{\frac{1}{2}}$

On a pris $\sigma_{\text {share }}=1$ et $\alpha=1$.

\subsubsection{Stratégie élitiste}

La stratégie élitiste utilisée est celle qui consiste à maintenir une population secondaire (archive) intégrée dans le processus d'optimisation. Les individus contenus dans cette archive participent aux opérations de sélection de croisement et de mutation. Pour maintenir sa taille constante, à chaque génération, les nouveaux individus non dominés remplacent les individus de l'archive qui les dominent.

\subsection{Résultats}

\subsubsection{Système linéaire}

Après l'exécution de l'algorithme, et pour un rayon de courbure $R_{\mathrm{c}}=500 \mathrm{~m}$, on obtient les solutions optimales qui forment le front de Pareto (Fig. 20).

Pour toutes les solutions non dominées, il n'y a pas de contact entre le boudin de la roue et le rail (Fig. 21). La contrainte sur $y_{11}^{*}$ du système (27) est satisfaite.

La meilleure solution est celle qui donne une vitesse critique de $59 \mathrm{~m} . \mathrm{s}^{-1}\left(212 \mathrm{~km} . \mathrm{h}^{-1}\right)$ et un déplacement de 9,99 mm. Mais, pour cette solution, le glissement n'est pas évité car :

$F R=1,8 \times 10^{4} N>\mu N=1,46 \times 10^{4} N$

La meilleure solution, pour laquelle on n'a ni contact entre le boudin de la roue et le rail, ni glissement, est celle qui donne une vitesse critique de $56 \mathrm{~m} . \mathrm{s}^{-1}\left(201 \mathrm{~km} \cdot \mathrm{h}^{-1}\right)$ 


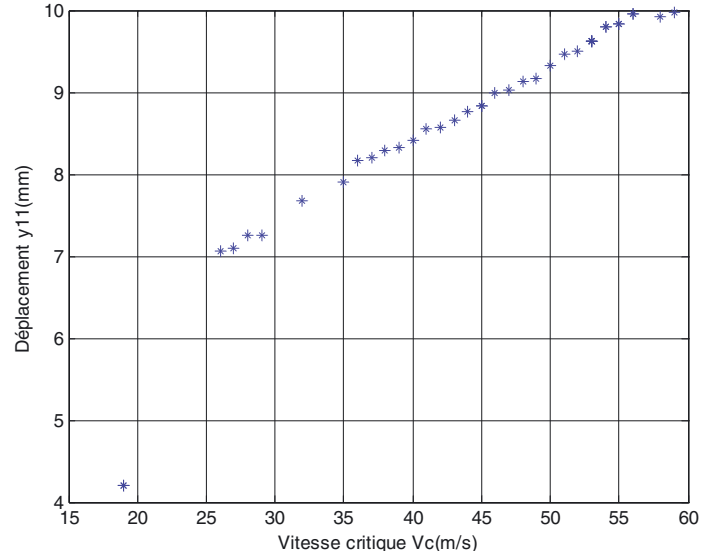

Fig. 21. Déplacement transversal total correspondant aux solutions optimales $\left(R_{\mathrm{c}}=500 \mathrm{~m}\right)$.

Tableau 5. Valeurs optimales des paramètres de construction du véhicule $\left(R_{\mathrm{c}}=500 \mathrm{~m}\right)$.

\begin{tabular}{|l|l|}
\hline$K_{x}\left(\mathrm{~N} \cdot \mathrm{m}^{-1}\right)$ & $1,91 \times 10^{6}$ \\
\hline$K_{y}\left(\mathrm{~N} \cdot \mathrm{m}^{-1}\right)$ & $5 \times 10^{7}$ \\
\hline $\bar{K}_{x}\left(\mathrm{~N} \cdot \mathrm{m}^{-1}\right)$ & $1,96 \times 10^{5}$ \\
\hline$\gamma_{\mathrm{e}}$ & 0,1 \\
\hline$a(\mathrm{~m})$ & 1,47 \\
\hline$M_{\mathrm{c}}(\mathrm{kg})$ & 45000 \\
\hline$M_{\mathrm{b}}(\mathrm{kg})$ & 3017 \\
\hline $\boldsymbol{V}_{\mathrm{c}}\left(\mathbf{k m} \cdot \mathbf{h}^{-1}\right)$ & $\mathbf{2 0 1}$ \\
\hline $\boldsymbol{y}_{11}(\mathbf{m m})$ & $\mathbf{9 , 9 5}$ \\
\hline
\end{tabular}

et un déplacement total de 9,95 $\mathrm{mm}$. Les valeurs des paramètres de construction du véhicule correspondant à cette solution sont illustrées dans le tableau 5 .

\subsubsection{Système non-linéaire}

Pour les rayons de courbure de $100 \mathrm{~m}$ et $150 \mathrm{~m}$, on ne peut pas éviter le contact entre le boudin de la roue et le rail. Alors pour ce rayon, on doit chercher les solutions du système (27) (sans contrainte sur le déplacement) pour lesquelles le véhicule ne déraille pas lors de sa circulation en courbe. La figure 22 montre le front de Pareto formé par les solutions optimales pour un rayon de courbure de $100 \mathrm{~m}$.

La figure 23 montre que pour toutes les solutions optimales le déplacement total dépasse le jeu entre le boudin de la roue et le rail.

Pour les rayons de courbure de 100 et $150 \mathrm{~m}$, les valeurs optimales des paramètres de construction, pour lesquelles le véhicule ne déraille pas, sont illustrées dans le tableau 6.

Pour des rayons de courbure de 200 à $350 \mathrm{~m}$, on peut trouver des solutions pour lesquelles on n'a ni contact ni glissement des roues par rapport aux rails, mais le véhicule est toujours instable lors de sa circulation en

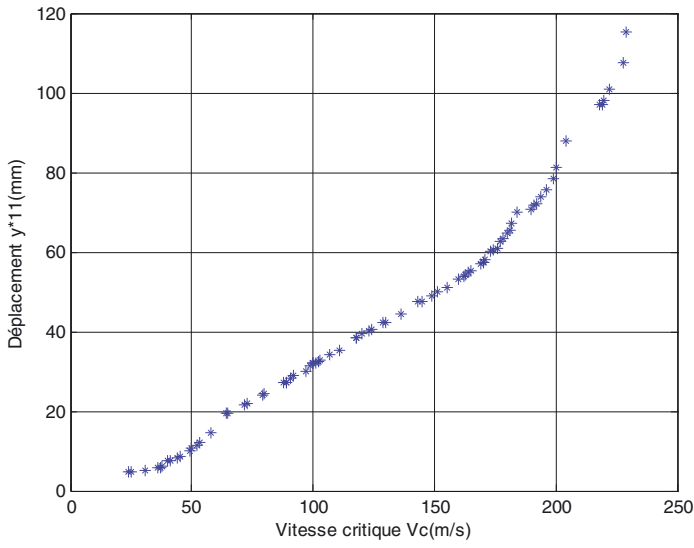

Fig. 22. Solutions optimales $\left(R_{\mathrm{c}}=100 \mathrm{~m}\right)$.

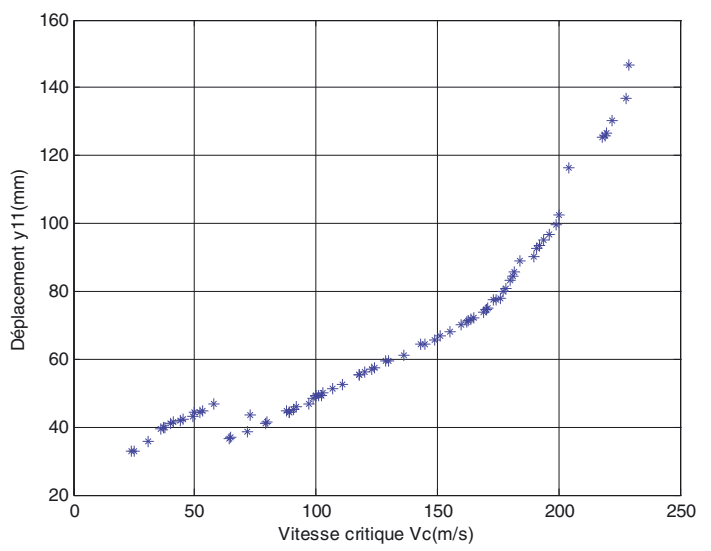

Fig. 23. Déplacement transversal total correspondant aux solutions optimales $\left(R_{\mathrm{c}}=100 \mathrm{~m}\right)$.

Tableau 6. Valeurs optimales des paramètres de construction du véhicule.

\begin{tabular}{|l|l|l|}
\hline $\boldsymbol{R}_{c}(\mathbf{m})$ & $\mathbf{1 0 0}$ & $\mathbf{1 5 0}$ \\
\hline$K_{x}\left(\mathrm{~N} \cdot \mathrm{m}^{-1}\right)$ & $1,15 \times 10^{6}$ & $1,4 \times 10^{6}$ \\
\hline$K_{y}\left(\mathrm{~N} \cdot \mathrm{m}^{-1}\right)$ & $5 \times 10^{7}$ & $3,35 \times 10^{7}$ \\
\hline $\bar{K}_{x}\left(\mathrm{~N} \cdot \mathrm{m}^{-1}\right)$ & $10^{5}$ & $1,45 \times 10^{5}$ \\
\hline$\gamma_{\mathrm{e}}$ & 0,1 & 0,1 \\
\hline$a(\mathrm{~m})$ & 1,48 & 1,46 \\
\hline$M_{\mathrm{c}}(\mathrm{kg})$ & 44000 & 42000 \\
\hline$M_{\mathrm{b}}(\mathrm{kg})$ & 3207 & 3150 \\
\hline $\boldsymbol{V}_{\mathrm{c}}\left(\mathrm{km} \cdot \mathbf{h}^{-1}\right)$ & $\mathbf{1 3 3 , 2}$ & $\mathbf{1 7 2 , 8}$ \\
\hline $\boldsymbol{F}_{11} / \boldsymbol{N}$ & $\mathbf{1 , 0 3}$ & $\mathbf{1 , 2 1}$ \\
\hline$\frac{\operatorname{tg} \theta_{\max }-\mu}{1+\mu \operatorname{tg} \theta_{\max }}$ & $\mathbf{1 , 0 5}$ & $\mathbf{1 , 2 2}$ \\
\hline
\end{tabular}

alignement. Ces solutions sont rejetées. Pour un rayon de $400 \mathrm{~m}$, les solutions optimales du système (27) sont montrées dans la figure 24 .

De même, pour toutes les solutions non dominées, il n'y a pas de contact entre le boudin de la roue et le rail (Fig. 25). La contrainte sur $y_{11}^{*}$ du système (27) est satisfaite. 


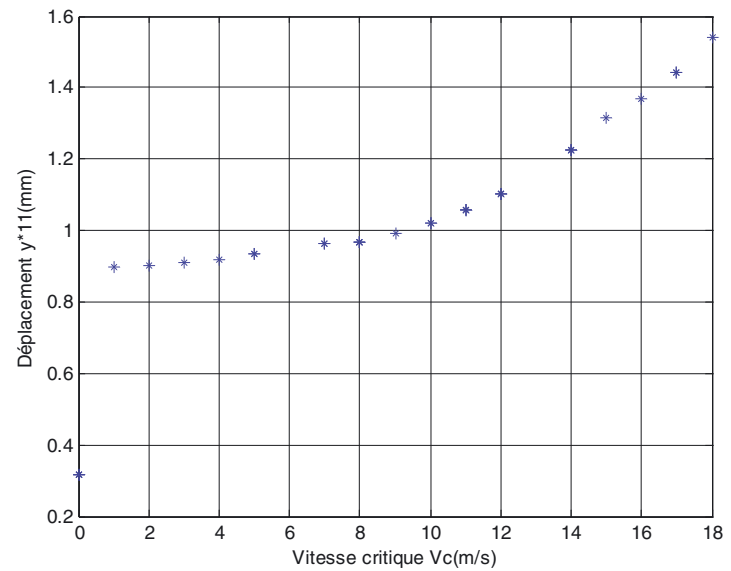

Fig. 24. Solutions optimales $\left(R_{\mathrm{c}}=400 \mathrm{~m}\right)$.

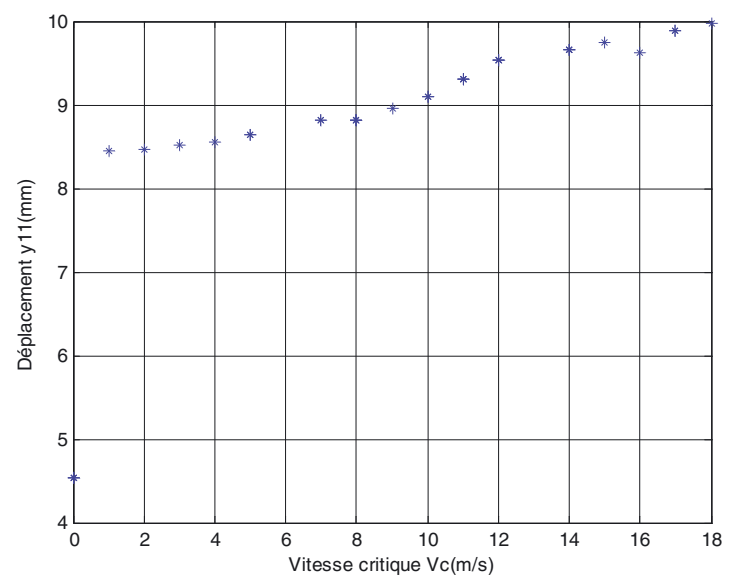

Fig. 25. Déplacement transversal total correspondant aux solutions optimales $\left(R_{\mathrm{c}}=400 \mathrm{~m}\right)$.

Pour toutes les solutions on a roulement sans glissement. La meilleure solution est celle qui donne une vitesse critique de $18 \mathrm{~m} . \mathrm{s}^{-1}\left(65 \mathrm{~km} \cdot \mathrm{h}^{-1}\right)$ et un déplacement de $1,47 \mathrm{~mm}$. Pour cette solution on a :

$y_{11}<10 \mathrm{~mm}$ et $F R=0,61 \times 10^{4} N<\mu N=1,32 \times 10^{4} N$.

Pour les rayons de courbure de $400 \mathrm{~m}$, les valeurs optimales des paramètres de construction sont illustrées dans le tableau 7.

\section{Conclusion}

L'utilisation des algorithmes génétiques est très efficace pour l'optimisation simple et multi-objective. En effet, pour l'optimisation simple, on a montré que cette méthode permet toujours de converger vers l'optimum global d'une fonction fortement discontinue et présentant plusieurs optima locaux. Aussi, elle nous a permis de trouver les valeurs optimales de plusieurs variables (paramètres de construction du véhicule) à la fois. Pour l'optimisation de la stabilité transversale en alignement, ces valeurs permettent au véhicule de circuler avec une très
Tableau 7. Valeurs optimales des paramètres de construction du véhicule $\left(R_{\mathrm{c}}=400 \mathrm{~m}\right)$.

\begin{tabular}{|l|l|}
\hline $\boldsymbol{R}_{c}(\mathbf{m})$ & $\mathbf{4 0 0}$ \\
\hline$K_{x}\left(\mathrm{~N} \cdot \mathrm{m}^{-1}\right)$ & $10^{6}$ \\
\hline$K_{y}\left(\mathrm{~N} \cdot \mathrm{m}^{-1}\right)$ & $5 \times 10^{7}$ \\
\hline $\bar{K}_{x}\left(\mathrm{~N} \cdot \mathrm{m}^{-1}\right)$ & $10^{5}$ \\
\hline$\gamma_{\mathrm{e}}$ & 0,1 \\
\hline$a(\mathrm{~m})$ & 1,12 \\
\hline$M_{\mathrm{c}}(\mathrm{kg})$ & 30206 \\
\hline$M_{\mathrm{b}}(\mathrm{kg})$ & 3500 \\
\hline $\boldsymbol{V}_{\mathrm{c}}\left(\mathrm{km} \cdot \mathbf{h}^{-1}\right)$ & $\mathbf{6 5}$ \\
\hline $\left.\boldsymbol{F R} \mathbf{( 1 0 ^ { 4 }} \mathbf{N}\right)$ & $\mathbf{0 , 6 1}$ \\
\hline$\mu \boldsymbol{N}\left(\mathbf{1 0}^{\mathbf{4}} \mathbf{N}\right)$ & $\mathbf{1 , 3 2}$ \\
\hline
\end{tabular}

grande vitesse, en toute sécurité. Pour l'optimisation de son comportement en courbe, les valeurs optimales des paramètres de construction permettent au véhicule de rouler sans glisser, et sans contact entre le boudin de la roue et le rail pour les grands et les faibles rayons de courbure.

Les résultats de l'optimisation simple ont montré que la majorité des valeurs optimales des paramètres de construction qui permettent une stabilité transversale en alignement du véhicule sont en contradiction avec celles trouvées pour l'optimisation de son comportement en courbe. Ce fait est parfaitement connu et confirmé par plusieurs travaux de recherches (cf. thèses H. Bettaieb, C. Pyrgidis). Dans cet article, on propose une méthode d'optimisation multi-objectifs utilisant la méthode des algorithmes génétiques, basée sur le principe de Pareto. Cette méthode a permis de trouver les valeurs optimales de plusieurs paramètres de construction du véhicule. Ces valeurs permettent au véhicule de circuler à une grande vitesse, en toute sécurité, dans une voie en alignement. Elles permettent aussi aux essieux d'avoir un déplacement minimum, qui évite le contact entre les boudins des roues et les rails et sans glissement entre la roue du véhicule et le rail lors de sa circulation en courbe de grands et de faibles rayons.

Les systèmes d'équations linéaires et non-linéaires ont permis de balayer tous les rayons de courbures $(100 \mathrm{~m} \leq$ $R_{\mathrm{c}} \leq 2000 \mathrm{~m}$ ). Pour les voies en courbe de rayon $R_{\mathrm{c}}$ inférieur à $500 \mathrm{~m}$, les résultats de l'optimisation multiobjective sont obtenus à partir du système non linéaire, tandis que, pour les rayons $R_{\mathrm{c}}$ supérieurs à $500 \mathrm{~m}$, les résultats sont obtenus à partir du système linéaire.

D'autres travaux de recherche supplémentaires pourraient être faits pour tester la robustesse de la méthode d'optimisation proposée dans cet article. Il serait utile aussi de tester le processus d'optimisation en utilisant de grands logiciels de dynamique comme Adams, Vampire ou Simpack. De même, il serait utile de tester d'autres modèles de contact roue-rail pour l'étude du comportement en courbe du véhicule. 


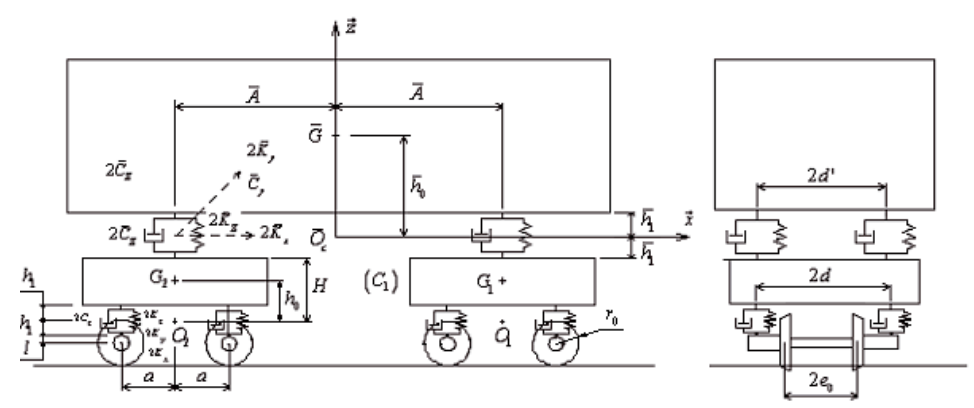

Fig. A.1. Véhicule.

\section{Annexe A}

\section{Paramètres d'inertie :}

$\begin{array}{ll}M_{\mathrm{c}}=43200 \mathrm{~kg} & : \text { Masse de la caisse } \\ \bar{r}_{x}=1,27 \mathrm{~m} & : \text { Rayon de giration de la caisse } \\ & \text { par rapport à } x \\ \bar{r}_{z}=7,5 \mathrm{~m} & : \text { Rayon de giration de la caisse } \\ & \text { par rapport à } z \\ M_{\mathrm{b}}=3020 \mathrm{~kg} & : \text { Masse du châssis de bogie } \\ r_{x}=0,84 \mathrm{~m} & : \text { Rayon de giration du châssis } \\ & \text { de bogie par rapport à } x \\ r_{y}=1,16 \mathrm{~m} & : \text { Rayon de giration du châssis } \\ & \text { de bogie par rapport à } y \\ r_{z}=1,16 \mathrm{~m} & : \text { Rayon de giration du châssis } \\ & \text { de bogie par rapport à } z \\ m_{1}=1500 \mathrm{~kg} & : \text { Masse d'un essieu } \\ m_{2}=250 \mathrm{~kg} & : \text { Masse des boîtes d'un essieu } \\ \rho_{x}=0,73 \mathrm{~m} & : \text { Rayon de giration de l'essieu } \\ & \text { par rapport à } x \\ \rho_{y}=0,25 \mathrm{~m} & : \text { Rayon de giration de l'essieu } \\ \rho_{z}=0,73 \mathrm{~m} & \text { par rapport à } y \\ & : \text { Rayon de giration de l'essieu } \\ & \text { par rapport à } z\end{array}$

Paramètres de la suspension secondaire :

$\begin{array}{ll}\bar{K}_{x}=1,73 \times 10^{5} \mathrm{~N} \cdot \mathrm{m}^{-1} & : \text { Rigidité } \\ & \text { longitudinale } \\ \bar{K}_{y}=1,73 \times 10^{5} \mathrm{~N} \cdot \mathrm{m}^{-1} & : \text { Rigidité transversale } \\ \bar{K}_{z}=5,3 \times 10^{5} \mathrm{~N} \cdot \mathrm{m}^{-1} & : \text { Rigidité verticale } \\ \bar{C}_{x}=0 \mathrm{~N} \cdot \mathrm{m}^{-1} & : \text { Amortissement } \\ & \text { longitudinal } \\ \bar{C}_{y}=3,5 \times 10^{4} \mathrm{~N} \cdot \mathrm{m}^{-1} & : \text { Amortissement } \\ & \text { transversal } \\ \bar{C}_{z}=1,5 \times 10^{4} \mathrm{~N} \cdot \mathrm{m}^{-1} & : \text { Amortissement } \\ & \text { vertical }\end{array}$

Paramètres de la suspension primaire :

$$
\begin{array}{ll}
K_{x}=4 \times 10^{7} \mathrm{~N} \cdot \mathrm{m}^{-1} & : \text { Rigidité longitudinale } \\
K_{y}=5 \times 10^{6} \mathrm{~N} \cdot \mathrm{m}^{-1} & : \text { Rigidité transversale } \\
K_{z}=9,75 \times 10^{5} \mathrm{~N} \cdot \mathrm{m}^{-1} & : \text { Rigidité verticale } \\
C_{x}=0 \mathrm{~N} \cdot \mathrm{m}^{-1} & : \text { Amortissement } \\
& \text { longitudinal } \\
C_{y}=0 \mathrm{~N} \cdot \mathrm{m}^{-1} & : \text { Amortissement } \\
& \text { transversal } \\
C_{z}=9910 \mathrm{~N} \cdot \mathrm{m}^{-1} & : \text { Amortissement } \\
& \text { vertical }
\end{array}
$$

\section{Paramètres géométriques :}

$$
\begin{aligned}
& \bar{h}_{0}=0,88 \mathrm{~m} \quad \text { : Distance verticale entre la } \\
& \bar{h}_{1}=0,21 \mathrm{~m} \quad \text { : Distance verticale entre la }
\end{aligned}
$$


$2 a=3 \mathrm{~m} \quad:$ Empattement du bogie

$2 l=0,165 \mathrm{~m} \quad$ : Distance verticale entre

la suspension primaire et

le centre d'inertie de l'essieu

\section{Paramètres géométriques du contact rail-roue :}

$$
\begin{aligned}
& \gamma_{\mathrm{e}}=0,1 \quad: \text { Conicité équivalente } \\
& \gamma_{0}=0,025 \quad \text { : Inclinaison du plan tangent } \\
& \text { du contact rail-roue par rapport à } \\
& \text { l'horizontale } \\
& r_{0}=0,45 \mathrm{~m} \quad \text { : Rayon du cercle de roulement } \\
& \text { de l'essieu en position centrée } \\
& \mathrm{e}_{0}=0,75 \mathrm{~m} \quad \text { : Demi-distance entre les points } \\
& \text { de contact rail-roue en position } \\
& \text { centrée } \\
& R=0,52 \mathrm{~m} \quad \text { : Rayon de courbure du profil } \\
& \text { de la roue } \\
& R^{\prime}=0,3 \mathrm{~m} \quad \text { : Rayon de courbure du profil } \\
& \text { du rail }
\end{aligned}
$$

\section{Références}

[1] P.J. Vermeulen, K.L. Johnson, Contact of non spherical, elastic bodies transmitting, tangential forces, ASME J. Appl. Mech. 11 (1964) 338-340

[2] Y. He, J. McPhee, Optimization of the lateral stability of rail vehicles, Vehicle System Dynamics 38 (2002) 361-390

[3] E. Lutton, Darwinisme artificiel : une vue d'ensemble, février 2004

[4] D.E. Goldberg, Genetic algorithms in search, optimization, and machine learning, Reading, MA: AddisonWesley, 1989

[5] J.R. Koza, Genetic Programming II: Automatic Discovery of Reusable Programs, Massachusetts Institute of Technolgy, 1994
[6] C.R. Houck, J.A. Joines, M.G. Kay, A genetic algorithm for function optimization: A Matlab implementation, NCSU-IE Technical Report 96-01, 1996

[7] E.K. Burke, J.D. Landa Silva, The influence of the fitness evaluation method on the performance of multi-objective search algorithms, Eur. J. Operational Res. 169 (2006) 875-897

[8] E. Zitzler, K. Deb, L. Thiele, Comparison of multiobjective evolutionary algorithms: empirical results (revised version), Technical report 70, Computer engineering and Networks Laboratory ETH Zurich, December 1999

[9] J. Horn, N. Nafpliotis, D.E. Goldberg, A Niched Pareto genetic algorithm for multi-objective optimization, IEEE Service Center 1 (1994) 82-87

[10] M. Laumanns, E. Zitzler, L. Thiele, A unified model for multi-objective evolutionary algorithms with elitism, in Congress on evolutionary computed Piscatcuna NJ, (2000) 46-53

[11] M. Laumanns, E. Zitzler, L. Thiele, On the effects of archiving, elitism, and density based selection in evolutionary multi-objective optimization, 2001

[12] H. Bettaieb, Étude de la stabilité transversale et le comportement en courbe d'un véhicule ferroviaire, Doctorat $3^{\text {e }}$ cycle mécanique appliqué à la construction, Université de Paris 6, 1983

[13] M. Ahmadian, S. Yang, Hopf bifurcation and hunting behaviour in a rail wheel set with flange contact, Nonlinear Dynamics 15 (1998) 15-30

[14] A. Mohan, Nonlinear investigation of the use of controllable primary suspensions to improve hunting in railway vehicles, Doctorat Thesis, Virginia, June 2003

[15] J. Elkins, H. Wu, Angle of attack and distance based criteria for flange climb derailment, Vehicle system Dynamics Supplement 33 (1999) 293-305

[16] H. Bettaieb, A. Mlika, Critères de choix des caractéristiques d'un véhicule ferroviaire circulant dans une courbe de faible rayon, Les Annales Maghrébines de l'Ingénieur 5 (1991) 195-210

[17] J.J. Kalker, Eon the rolling contact of two elastic bodies in the presence of dry friction, Ph.D. thesis, Delft university of technology, 1967 